DOE/ER/40561-307-INT96-21-004

\title{
Some implications of meson dominance in weak interactions
}

\author{
Peter Lichard \\ Department of Theoretical Physics, Comenius University, 842-15 Bratislava, Slovak Republic \\ and Institute of Physics, Silesian University, 746-01 Opava, Czech Republic \\ and Institute for Nuclear Theory, University of Washington, Seattle, WA 98195
}

\begin{abstract}
The hypothesis is scrutinized that the weak interaction of hadronic systems at low energies is dominated by the coupling of the pseudoscalar, vector, and axial-vector mesons to the weak gauge bosons. The strength of the weak coupling of the $\rho(770)$ meson is uniquely determined by vector-meson dominance in electromagnetic interactions; flavor and chiral symmetry breaking effects modify the coupling of other vector mesons and axial-vector mesons. Many decay rates are calculated and compared to experimental data and partly to predictions of other models. A parameter-free description of the decay $K^{+} \rightarrow \pi^{+} \ell^{+} \ell^{-}$is obtained. Predictions for several not yet observed decay rates and reaction cross sections are presented. The relation between the conserved vector current hypothesis and meson dominance is clarified. Phenomenological success of the meson dominance suggests that in some calculations based on the standard model the weak quark-antiquark annihilation and creation diagrams may be more important than anticipated so far. The processes are identified where the meson dominance fails, implying that they are governed, on the quark level, by some other standard model diagrams.
\end{abstract}

PACS number(s): 12.15.-y, 12.15.Ji, 12.40.Vv 


\section{INTRODUCTION}

The idea of vector meson dominance (VMD), which was proposed a long time ago [1], has proven to be very fruitful in describing the electromagnetic interactions of hadrons at low energies. It is routinely used, even today when the standard theory [2, 3] provides a unified picture of all interactions among leptons and quarks. The reason for the present day popularity of effective theories is the difficulty encountered when building a bridge between the world of quarks and gluons and that of hadrons.

According to the VMD hypothesis the electromagnetic interactions of hadrons are mediated by neutral vector mesons $\left(\rho^{0}, \omega, \phi\right.$, and to a lesser extent also their higher recurrences) which couple to the electromagnetic field $a_{\mu}$ according to the Lagrangian

$$
\mathcal{L}_{\mathrm{VMD}}=-e \frac{m_{\rho}^{2}}{g_{\rho}}\left(V_{\rho^{0}}^{\mu}+\frac{1}{3} V_{\omega}^{\mu}-\frac{\sqrt{2}}{3} V_{\phi}^{\mu}\right) a_{\mu},
$$

where $V$ 's are vector meson field operators. The presence of the ratio of the $\rho$ mass squared to the $\rho \pi \pi$ coupling constant $g_{\rho}\left(g_{\rho}^{2}=36.56 \pm 0.29\right)$ is required by the normalization condition $F_{\pi}(0)=1$ for the pion form factor. The other factors follow from invariance under the $U$ spin $S U(2)$ subgroup of the (flavor) $S U(3)$ group and the assumption that the physical $\omega$ meson does not contain $s$-quarks.

The idea of the universality of the vector current led very soon to the application of VMD in weak interactions. The early development was reviewed in [4.5]. But, unlike in QED, the transition amplitudes between the weak gauge bosons and mesons are nonvanishing also for pseudoscalar and axial-vector mesons. It is therefore natural to generalize the VMD and assume that the weak interaction of hadronic systems is dominated by the coupling of pseudoscalar, vector, and axial-vector mesons to the gauge bosons $W^{ \pm}$and $Z^{0}$. We will refer to this hypothesis as meson dominance (MD).

Qualitative support for MD in weak interactions comes at least from two sources: (1) Individual pseudoscalar, vector and axial-vector mesons are copiously produced in the decay of the tau lepton; (2) MD naturally explains why the ratios among various charge configurations of hadronic final states in weak decays often follow the rules implied by isospin invariance, which is otherwise violated in weak interactions.

The MD hypothesis has two components. Firstly, the assumption that the weak interaction of hadronic systems is dominated by the coupling of individual mesons to the weak gauge bosons means, on the quark level, restriction to a certain class of perturbative expansion diagrams. This class does not include, e.g., the penguin and box diagrams. Secondly, in order to make the MD a quantitative concept we have to establish the effective Lagrangian of the interaction between mesons and gauge bosons. This will be done in Sec. [I].

The question arises whether we really need a simple and approximate phenomenological approach to the electroweak interaction since we believe that the fundamental theory exists, which allows one to calculate everything from first principles. We think that the reasons for exploring MD are twofold.

It is true that the basic electroweak diagrams of most decay modes are relatively simple. But, as a matter of fact, the QCD effects play an important role. The calculation of QCD corrections to the basic electroweak diagrams is the most difficult and involved part [6]. 
In contrary, the MD approach takes advantage of the fact that the Mother Nature made some QCD calculations for us, even nonperturbatively, when she built hadrons. It is not true in general. The results of some QCD calculations (e.g., QCD penguin diagrams, QCD corrections to the weak and electromagnetic penguins and to the box diagrams) are not accessible so easily and MD cannot be explored in such cases. Anyhow, an approximately correct description of some process by the MD may have heuristic value for the more fundamental approaches by showing which quark diagrams may be most important. We will try to illustrate this point in Sec. V.

Another reason for our considering the MD approach is the relations between theory and experiment. The fundamental theories or models more sophisticated than MD do not often provide simple formulas for various distributions that would be suitable for use by experimentalists to fit their data. As a consequence, formulas lacking dynamic motivation or sometimes even violating the basic principles of quantum mechanics are used. The MD approach may be able to offer formulas, even if approximate, that are simple and reflect, at least in a crude way, the underlying dynamics.

We will try to keep this paper selfcontained and provide all the formulas and numbers we used. The values of input parameters (masses, hadronic widths, lifetimes) needed in our calculations were taken from [7]. Unless stated otherwise, so were the experimental values of branching fractions to which we compare our results. The quoted errors of our results reflect only those of input parameters. No attempt has been made to assess the systematic uncertainties of the meson dominance approach. Not to expand the scope of the paper too much, we do not compare, with a few exceptions, our results to those of existing models. The references to the latter can be traced back from the most topical ones [8,9], some of them are mentioned in this paper later on.

Throughout the paper $P, S, V$, and $A$ will be used as generic labels for pseudoscalar, scalar, vector, and axial-vector mesons, respectively. In Lagrangians, the field operators will always be those of individual members of isospin multiplets. The isospin symmetry of hadronic Lagrangians will be ensured through the relations among the coupling constants for different charge combinations of participating mesons.

In the next section, we write effective Lagrangians describing the coupling of vector and axial-vector mesons to charged and neutral weak gauge bosons $W^{ \pm}$and $Z^{0}$ and define the parameters of our approach. Section IIT deals with the decays of the $\tau$ lepton, some used as a source of information about the MD parameters. In Sec. IV, we investigate vector current processes in which the core of hadronic part is the $P P V$ vertex, with vector meson $V$ converting into the charged gauge boson. They include the following types of decays: $P_{1} \rightarrow P_{2}+\ell \nu_{\ell}, P_{1} \rightarrow P_{2}+P_{3}, P_{1} \rightarrow P_{2}+V$, and $P_{1} \rightarrow P_{2}+A$. We calculate also the cross sections of the antineutrino-electron and meson-electron binary reactions that are related to the semileptonic decay shown above by crossing symmetry. In Section $\square$ we show that MD leads to a parameter-free formula for the rate of the decay $K^{+} \rightarrow \pi^{+} e^{+} e^{-}$that agrees with the experimental value. Predictions for the dimuon mode and for the transitions of $D^{+}$and $D_{s}$ mesons into the same final state are also made. Section $\left.\nabla\right]$ is devoted to the relation between the conserved vector current (CVC) and MD hypotheses. We summarize our main points and add a few comments in Sec. VII. Some related issues are deffered to the Appendices. In Appendix A we extract some hadronic coupling constants from data on hadronic and radiative decay widths. Appendix B shows the decay rate of $P_{1} \rightarrow P_{2}+\ell \nu_{\ell}$ 
for arbitrary form factors, which was used in Sec. V1.

\section{DEFINING MESON DOMINANCE IN WEAK INTERACTIONS}

In this section we will use plausible arguments based on the standard model Lagrangian and the VMD in electromagnetic sector in order to find the effective Lagrangian that describes the coupling of vector and axial-vector mesons to the weak gauge bosons, both charged and neutral. In a search for it we first discuss the dynamical content of the VMD in electromagnetic interactions from the quark model point of view. Then we will apply the same procedure to the weak interactions.

\section{A. Vector meson dominance in electromagnetic interactions}

The electromagnetic part of the standard model Lagrangian

$$
\mathcal{L}_{\mathrm{EM}}(x)=-j^{\mu}(x) a_{\mu}(x)
$$

contains the electromagnetic field operator $a_{\mu}$ and the quark electromagnetic current

$$
j^{\mu}(x)=e \sum_{i=1}^{3}\left[\frac{2}{3} \bar{u}_{i}(x) \gamma^{\mu} u_{i}(x)-\frac{1}{3} \bar{d}_{i}(x) \gamma^{\mu} d_{i}(x)\right],
$$

where $u_{i}\left(d_{i}\right)$ denotes the field operator of the up (down) quark from the $i$ th generation. The matrix element of an electromagnetic process with a $\rho^{0}$ in the initial state will contain the factor

$$
\left\langle 0\left|j^{\mu}(0)\right| p, \lambda\right\rangle_{\rho^{0}}=\frac{2}{3} e\left\langle 0\left|\bar{u}(0) \gamma^{\mu} u(0)\right| p, \lambda\right\rangle_{\rho^{0}}-\frac{1}{3} e\left\langle 0\left|\bar{d}(0) \gamma^{\mu} d(0)\right| p, \lambda\right\rangle_{\rho^{0}},
$$

where $p$ and $\lambda$ are the four-momentum and polarization of $\rho^{0}$, respectively. Only quarks of the first generation matter. The matrix elements must transform like four-vectors. The only two four-vectors we have at our disposal are the four-momentum $p^{\mu}$ of the $\rho^{0}$ and its polarization vector $\epsilon^{\mu}(p, \lambda)$. Because we are interested in low energy interactions, we will neglect the term proportional to the four-momentum. We thus write

$$
\left\langle 0\left|\bar{u}(0) \gamma^{\mu} u(0)\right| p, \lambda\right\rangle_{\rho^{0}}=F_{\rho} \epsilon^{\mu}(p, \lambda),
$$

where $F_{\rho}$ is a constant. Isospin invariance together with the isovector character of the $\rho$ implies that

$$
\left\langle 0\left|\bar{d}(0) \gamma^{\mu} d(0)\right| p, \lambda\right\rangle_{\rho^{0}}=-\left\langle 0\left|\bar{u}(0) \gamma^{\mu} u(0)\right| p, \lambda\right\rangle_{\rho^{0}} .
$$

Putting it into (2.2) and using Eq. (2.3) we get

$$
\left\langle 0\left|j^{\mu}(0)\right| p, \lambda\right\rangle_{\rho^{0}}=e F_{\rho} \epsilon^{\mu}(p, \lambda) .
$$

On the other hand, we have the relation 


$$
\left\langle 0\left|V_{\rho^{0}}^{\mu}(0)\right| p, \lambda\right\rangle_{\rho^{0}}=\mathcal{N} \epsilon^{\mu}(p, \lambda) .
$$

Both $F_{\rho}$ and the constant $\mathcal{N}$ depend on the normalization of one-particle states, but their ratio does not. Comparing (2.4) and (2.5) we see that the Lagrangian obtained from (2.1) by the substitution

$$
j^{\mu}(x) \rightarrow e \frac{F_{\rho}}{\mathcal{N}} V_{\rho^{0}}^{\mu}(x)
$$

gives the same low energy matrix element as the original Lagrangian (2.1). In order the get correctly the first term in Eq. (1.1), which is fixed by the normalization of the pion form factor, the following relation must be held

$$
\frac{F_{\rho}}{\mathcal{N}}=\frac{m_{\rho}^{2}}{g_{\rho}}
$$

Repeating all the steps for the isoscalar $\omega$ and $\phi$ mesons, and using $S U(3)$ invariance relations

$$
\left\langle 0\left|\bar{u}(0) \gamma^{\mu} u(0)\right| p, \lambda\right\rangle_{\omega}=\frac{1}{\sqrt{2}}\left\langle 0\left|\bar{s}(0) \gamma^{\mu} s(0)\right| p, \lambda\right\rangle_{\phi}=\left\langle 0\left|\bar{u}(0) \gamma^{\mu} u(0)\right| p, \lambda\right\rangle_{\rho^{0}},
$$

together with (2.6), we obtain the other terms of Eq. (1.1). We assumed that the $\phi$ meson transforms like a pure $\bar{s} s$ state.

We consider it important to stress that $g_{\rho}$ in Eq. (1.1) and, as a consequence, also in the weak Lagrangians we are going to introduce in what follows, is the coupling constant of the $\rho \pi \pi$ interaction, determined from the $\rho \rightarrow \pi \pi$ decay width. This is strictly required by the condition $F_{\pi}(0)=1$ on the electromagnetic form factor of the $\pi^{+}$meson calculated from (11.1). In some papers this important constrain was ignored and the $g_{\rho}$ was determined from the electronic decay width of the $\rho^{0}$ meson. It is true that if one calculates the rate of the $\rho^{0} \rightarrow e^{+} e^{-}$decay from the Lagrangian (1.1) with $g_{\rho}$ determined from $\rho \rightarrow \pi \pi$, the result is obtained which is smaller by a factor of $1.45 \pm 0.07$ than the experimental value. But this is, as we will show elsewhere, the effect of higher $\rho$ resonances. It is improper to mimic this effect by violating the normalization condition of the form factor.

Also, if higher mesons from the $\rho$ family are added into (1.1), the coupling of $\rho(770)$ to the electromagnetic field may be modified. The normalization condition for the pion form factor implies a definite relation among $\rho \gamma$ coupling constants. The safest way of accounting for the influence of higher $\rho$ resonances is to replace the properly normalized form factor induced by $\rho(770)$ with a properly normalized form factor containing all considered resonances. Preference is for the experimentally determined ones, if available.

\footnotetext{
${ }^{1}$ Relations (2.7) and other of this kind stem from the transformation properties of the wave functions and field operators, and do not mean that we ignore the gluon or sea quark content of the meson wave functions.
} 


\section{B. Meson dominance in weak interactions}

Now we are going to apply the same procedure to the weak interactions. First we will fix the coupling of the charged $\rho$ mesons to the charged weak gauge bosons $W^{ \pm}$. We start again from the standard model Lagrangian, this time from the part that exhibits the charged current weak interaction of the $u$ and $d$ quarks,

$$
\begin{aligned}
\mathcal{L}_{u d} & =-W_{\mu}^{-} j_{u d}^{\mu}+\text { h.c. } \\
j_{u d}^{\mu} & =\frac{g}{2 \sqrt{2}} V_{u d} \bar{d} \gamma^{\mu}\left(1-\gamma_{5}\right) u,
\end{aligned}
$$

where $g=e / \sin \theta_{W}$ is the electroweak coupling constant and $V_{u d}$ is the relevant element of the Cabibbo-Kobayashi-Maskawa (CKM) matrix [10,11]. The matrix element for a process with a $\rho^{+}$in the initial state is proportional to

$$
\left\langle 0\left|j_{u d}^{\mu}(0)\right| p, \lambda\right\rangle_{\rho^{+}}=\frac{g}{2 \sqrt{2}} V_{u d}\left\langle 0\left|\bar{d}(0) \gamma^{\mu}\left(1-\gamma_{5}\right) u(0)\right| p, \lambda\right\rangle_{\rho^{+}}
$$

The axial-vector part does not contribute, and for the vector part we can write

$$
\left\langle 0\left|\bar{d}(0) \gamma^{\mu} u(0)\right| p, \lambda\right\rangle_{\rho^{+}}=\sqrt{2}\left\langle 0\left|\bar{u}(0) \gamma^{\mu} u(0)\right| p, \lambda\right\rangle_{\rho^{0}}=\sqrt{2} F_{\rho} \epsilon^{\mu}(p, \lambda) .
$$

Writing an equation analogous to (2.5), and using the value of $F_{\rho} / \mathcal{N}$ as implied by the VMD for electromagnetic interactions, see (2.6), we come to the conclusion that the effective Lagrangian for the low energy weak interaction of the charged $\rho$ mesons is

$$
\mathcal{L}_{\rho^{ \pm}}=-\frac{g m_{\rho}^{2}}{2 g_{\rho}} V_{u d} W_{\mu}^{-} V_{\rho^{+}}^{\mu}+\text { h.c. }
$$

Now let us investigate the coupling of the $K^{*+}$ to $W^{+}$. The corresponding piece of the standard model Lagrangian is

$$
\begin{aligned}
\mathcal{L}_{u s} & =-W_{\mu}^{-} j_{u s}^{\mu}+\text { h.c. } \\
j_{u s}^{\mu} & =\frac{g}{2 \sqrt{2}} V_{u s} \bar{s} \gamma^{\mu}\left(1-\gamma_{5}\right) u .
\end{aligned}
$$

In analogy with (2.9) we define the constant $F_{K^{*}}$ by the relation

$$
\left\langle 0\left|\bar{s}(0) \gamma^{\mu} u(0)\right| p, \lambda\right\rangle_{K^{*+}}=\sqrt{2} F_{K^{*}} \epsilon^{\mu}(p, \lambda) .
$$

Equation (2.5) is valid also for $K^{*+}$ with the same value of the normalization constant $\mathcal{N}$. Using (2.6) and defining

$$
w_{K^{*}}=\frac{F_{K^{*}}}{F_{\rho}} \frac{m_{\rho}^{2}}{m_{K^{*+}}^{2}}
$$

we arrive at the conclusion that the Lagrangian

$$
\mathcal{L}_{K^{* \pm}}=-\frac{g m_{K^{*+}}^{2}}{2 g_{\rho}} w_{K^{*}} V_{u s} W_{\mu}^{-} V_{K^{*+}}^{\mu}+\text { h.c. }
$$


gives the same values of all observables in low energy processes with a $K^{* \pm}$ in the initial or final state as the standard model Lagrangian (2.10) after the constant $w_{K^{*}}$ is properly adjusted. In the $S U_{f}(3)$ symmetry was exact we would have $w_{K^{*}}=1$.

The case of the axial-vector meson $a_{1}$ can be handled in the same way. We define the constant $F_{a_{1}}$ by the relation

$$
\left\langle 0\left|\bar{u}(0) \gamma^{\mu} \gamma_{5} u(0)\right| p, \lambda\right\rangle_{a_{1}^{0}}=F_{a_{1}} \epsilon^{\mu}(p, \lambda) .
$$

With Eq. (2.6) in mind we further define

$$
w_{a_{1}}=\frac{F_{a_{1}}}{F_{\rho}} \frac{m_{\rho}^{2}}{m_{a_{1}}^{2}}
$$

The Lagrangian

$$
\mathcal{L}_{a_{1}^{ \pm}}=\frac{g m_{a_{1}}^{2}}{2 g_{\rho}} w_{a_{1}} V_{u d} W_{\mu}^{-} A_{a_{1}^{+}}^{\mu}+\text { h.c. }
$$

then leads to the same matrix elements for processes with the $a_{1}$ in the initial or final state as the original standard model Lagrangian (2.8). In that sense it represents an effective Lagrangian for the weak interaction of $a_{1}^{ \pm}$mesons. The constant $w_{a_{1}}$ is a phenomenological parameter of the MD approach and should be determined from data. In the chiral limit $m_{u}=m_{d}=0$ the $\rho$ and $a_{1}$ mesons constitute a parity degenerate doublet and the $w_{a_{1}}$ would be unity.

The $a_{1}$ meson belongs to the ${ }^{3} P_{1}$ octet of axial-vector mesons. There has not been any experimental indication that its counterpart from the ${ }^{1} P_{1}$ octet, namely, the $b_{1}$ axial-vector meson, also couples to the weak gauge bosons. For instance, it has not been identified among the decay products of the $\tau$ lepton. One possible explanation follows. The valence quark and antiquark in the $b_{1}$ form a singlet spin state. Their helicities in the meson rest frame thus tend to be equal, what leads to a small matrix element of the weak quark current. But the coupling of the $b_{1}$ to the gauge bosons, in other words, the existence of a second class axial-vector current [12], is not ruled out absolutely by the quark model approach. There is no obvious reason for the matrix element of the type (2.13) written for the $b_{1}$ meson to vanish identically.

To complete our considerations of the MD in the charged current weak interactions let us recall that quarks from higher generations enter the standard model Lagrangian in the same way as the $u$ and $d^{\prime}$ quarks from the first generation. This suggests that the most general form of the charged current MD is

$$
\mathcal{L}_{\text {c.c. }}=-\frac{g}{2 g_{\rho}} W_{\mu}^{-}\left[\sum_{V=\rho^{+}, K^{*+}, \ldots} m_{V}^{2} V_{V} w_{V} V^{\mu}-\sum_{A=a_{1}^{+}, K_{1}^{+}, \ldots} m_{A}^{2} V_{A} w_{A} A^{\mu}\right]+\text { h.c. }
$$

Here $V^{\mu}$ and $A^{\mu}$ are the field operators of positively charged vector $\left(\rho^{+}, K^{*+}, D^{*+}, D_{s}^{*+}\right.$, $B^{*+}$ ) and axial-vector mesons $\left[a_{1}^{+}, K_{1}(1400)^{+}, D_{1}^{+}, D_{s 1}^{+}\right]$, respectively. Unlisted states either do not exist or have not yet been discovered. $V_{V}$ and $V_{A}$ are the elements of the CKM matrix that correspond to the valence quark composition of the particular vector or axial-vector 
meson. To make Eq. (2.15) compact we have introduced $w_{\rho^{+}} \equiv 1$. In the case of exact $S U_{f}(6)$ symmetry also the other $w_{V}$ would be equal to one and all $w_{A}$ would be equal to $w_{a_{1}^{+}}$. The actual values may be different.

Anyhow, the $w_{A}$ should not differ too much from the $w_{V}$ of the vector mesons with the same flavor, because the corresponding vector and axial-vector mesons form a chiral symmetry doublet. The relative sign of the vector and axial-vector parts of (2.15) is important for processes to which they both contribute.

The parameters $w_{V}$ and $w_{A}$ enter formulas for observable quantities in combinations with other parameters (CKM matrix elements, strong interaction coupling constants). In some cases it makes their extraction from the data impossible. This does not diminish appreciably the predictive power of the MD because the same product of parameters determines the rates or cross sections of several processes. We can thus fix the normalization using one piece of data and other quantities then are predicted by the MD. This approach will be used extensively in Section [D.

In the cases when the vector or axial-vector meson that couples to the gauge boson appears as one of the final state particles it is useful to define the quantities

$$
Y_{M}=\left|w_{M} V_{M}\right|^{2}
$$

where $M$ stands for any of the charged vector and axial-vector mesons. $V_{M}$ is the element of the CKM matrix pertinent to the valence quark and antiquark of the particular meson, and $w_{M}$ is the parameter in the effective charged current MD Lagrangian (2.15) that appears in

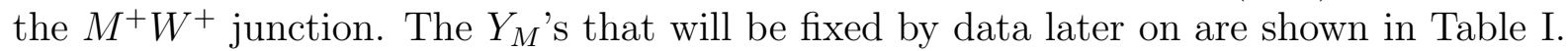

A few more words are needed about the strange axial-vector mesons that exist in two sorts, $K_{1}(1270)$ and $K_{1}(1400)$. Analysis of the branching fractions of the $\tau^{-}$lepton suggests that the coupling of $K_{1}(1400)$ to the weak gauge bosons is stronger than that of $K_{1}(1270)$. First of all, the $\tau^{-}$branching fraction to $K_{1}(1400)^{-}$is $(8 \pm 4) \times 10^{-3}$, to $K_{1}(1270)^{-},(4 \pm$ 4) $\times 10^{-3}$. Next, the branching ratios of $\tau^{-}$to $K^{-} \pi^{+} \pi^{-}, \bar{K}^{0} \pi^{-} \pi^{0}$, and $K^{-} \pi^{0} \pi^{0}$ systems are compatible with 4:4:1 ratio, which is typical for decay of an $I=1 / 2$ resonance $\left(K_{1}^{-}\right)$ to those systems through the $\left(K^{*} \pi\right)^{-}$intermediate state. It again points to $K_{1}(1400)$ with its branching fraction to $K^{*} \pi$ of $(94 \pm 6) \%$ rather than to $K_{1}(1270)$ [(16 \pm 5$\left.) \%\right]$. Finally, if the axial strange mesons that couple to the $W$ gauge boson were the ${ }^{3} P_{1}$ state $K_{1 A}$ or the ${ }^{1} P_{1}$ state $K_{1 B}$, nearly equal mixes of the $K_{1}(1270)$ and $K_{1}(1400)$, then the $K_{1}(1270)$ and its decay products would be more visible in $\tau$ decays. Also, in the recent work [13] current algebra was applied in the three-pseudoscalar-meson decays of the $\tau$ lepton. The $K^{-} \rho^{0} \nu_{\tau}$ mode [the dominant strong decay mode of $K_{1}(1270)$ ] was shown to be consistent with zero.

The higher vector and axial-vector recurrences are not explicitly shown in (2.15). Generally, their influence will be difficult to take into account due to insufficient knowledge of their couplings to other hadrons. In some cases (certainly in the phenomenologically most important case of the $\rho$ meson family) they can be taken into account by replacing a simple pole contribution, stemming from the virtual meson propagator, by an empirically determined electromagnetic form factor. When appropriate, we will use that of [14].

When using the same procedure to determine the coupling of the vector and axial-vector mesons to the $Z$ gauge boson, we find that only the truly neutral (all additive quantum numbers vanishing) mesons can couple. The coupling of those consisting of a down quark 
and a down antiquark from different generations is proportional to the off-diagonal elements of the product $V^{\dagger} V$ and is therefore forbidden by the unitarity of the CKM matrix $V$. The neutral mesons formed of valence up quark and antiquark from different generations are excluded by the form of the neutral weak current itself. The effective Lagrangian describing the interaction of truly neutral vector and axial-vector mesons with the $\mathrm{Z}$ boson has the form

$$
\mathcal{L}_{\text {n.c. }}=-\frac{g}{2 g_{\rho} \cos \theta_{W}} Z_{\mu}\left[\sum_{V=\rho^{0}, \omega, \cdots} m_{V}^{2} G_{V} V^{\mu}-\sum_{A=a_{1}^{0}, f_{1}, \cdots} m_{A}^{2} G_{A} A^{\mu}\right],
$$

where $G_{\rho^{0}}=w_{\rho^{0}}\left(1-2 \sin ^{2} \theta_{W}\right), G_{\omega}=-2 / 3 w_{\omega} \sin ^{2} \theta_{W}, G_{J / \psi}=\sqrt{2} w_{J / \psi}\left(1 / 2-4 / 3 \sin ^{2} \theta_{W}\right)$, and $G_{V}=\sqrt{2} w_{V}\left(-1 / 2+2 / 3 \sin ^{2} \theta_{W}\right)$ for $V=\phi, \Upsilon$. For axial-vector mesons we have $G_{a_{1}^{0}}=w_{a_{1}^{0}}, G_{A}=-w_{A} / \sqrt{2}$ for the pure $\bar{d} d$ states $\left[A=f_{1}(1510), \chi_{b 1}\right], G_{A}=w_{A} / \sqrt{2}$ for the pure $\bar{u} u$ states $\left(\chi_{c 1}\right)$, and $G_{A}=0$ for $f_{1}(1285)$, the isoscalar axial-vector counterpart of the $\omega(782)$. Most of the constants $w_{V}$ and $w_{A}$ represent new parameters, with values expected not to be far from unity. Isospin symmetry enables to relate some of them to the corresponding parameters of the charged current Lagrangian (2.15), e.g., $w_{\rho^{0}}=w_{\omega}=w_{\rho^{+}} \equiv$ $1, w_{a_{1}^{0}}=w_{a_{1}^{+}}$. Under exact $S U_{f}(3)$ symmetry we would also have $w_{\phi}=1$.

The weak interaction of pseudoscalar mesons is routinely described by the Lagrangian

$$
\mathcal{L}_{P}=-i \frac{g}{2 \sqrt{2}} W_{\mu}^{-} \sum_{P=\pi^{+}, K^{+}, \cdots} f_{P} V_{P} \partial^{\mu} \varphi_{P}+\text { h.c. }
$$

where $V_{P}$ is the element of the CKM matrix pertinent to valence quark and antiquark of the meson $P$ and $f_{P}$ is the pseudoscalar-meson decay constant defined for the $\pi^{+}$meson by

$$
\left\langle 0\left|\bar{d}(0) \gamma^{\mu} \gamma_{5} u(0)\right| p\right\rangle_{\pi^{+}}=i f_{\pi^{+}} p^{\mu}
$$

and analogously for other mesons. Observables (decay rates, cross sections) of the processes with the pseudoscalar meson $P$ either in the initial or final state will be proportional to the quantity

$$
Z_{P}=\left|f_{P} V_{P}\right|^{2}
$$

The values of these parameters for different pseudoscalar mesons can be determined from their leptonic branching fractions $P \rightarrow \ell \nu$ and are shown in Table 【I. In the case of the $\pi^{+}$ and $K^{+}$leptonic decays the radiative corrections are important. We used the prescription defined in Suzuki's article in [7], p. 319. For the $D^{+}$meson only an upper limit on the leptonic branching fraction is known experimentally. Here we used the recent lattice calculation [15] result $f_{D^{+}}=(208 \pm 35 \pm 12) \mathrm{MeV}$ and $\left|V_{c d}\right|=0.224 \pm 0.016$ from [7]. We summed the errors quadratically.

From the MD point of view the coupling of scalar mesons to weak gauge bosons is not excluded. However, the success of the conserved vector current (CVC) hypothesis shows

\footnotetext{
${ }^{2}$ In this paper we will not be faced with the necessity to consider the interference of several diagrams.
} 
that this coupling, which represents a second-class vector current [12], must be negligible. Nevertheless, let us define the scalar-meson decay constant of the $a_{0}^{+}$meson analogously to (2.19) by means of the matrix element of the vector part of the weak current

$$
\left\langle 0\left|\bar{d}(0) \gamma^{\mu} 0 u(0)\right| p\right\rangle_{a_{0}^{+}}=i f_{a_{0}^{+}} p^{\mu} .
$$

and similarly for other charged scalar mesons [in fact, apparently only one exists- $K_{0}(1430)$ ]. The effective Lagrangian is given as

$$
\mathcal{L}_{S}=i \frac{g}{2 \sqrt{2}} W_{\mu}^{-} \sum_{S=a_{0}^{+}, K_{0}^{+}} f_{S} V_{S} \partial^{\mu} \varphi_{S}+\text { h.c. . }
$$

In the next section we will show on the basis of experimental data that the decay constant of the $a_{0}^{+}$meson is at least twenty times smaller than that of the $\pi^{+}$.

In this paper we will not consider processes in which a truly neutral spin zero meson couples to the neutral weak gauge boson $Z$. We therefore do not write the corresponding Lagrangians here.

What we have done in this section can only be considered as a more or less educated guess, not derivation, of what the effective Lagrangians for the weak interaction of vector, axialvector, pseudoscalar, and scalar vectors may look like. Moreover, we have so far considered only real (incoming or outgoing) mesons. Going off mass-shell may, in principle, convert the Lagrangian parameters $w_{V}, w_{A}, f_{P}$, and $f_{S}$ into arbitrary functions of meson virtualities $p^{2}$. To proceed further we will neglect this possibility and postulate the validity of Lagrangians as given in Eqs. (2.15), (2.17), (2.18), and (2.22) for both real and virtual mesons. This postulate, together with the restriction to a certain class of quark diagrams, discussed in Section 【, constitute the main ingredients of the meson dominance in weak interactions.

\section{MESON DOMINANCE AND DECAY MODES OF THE $\tau$ LEPTON}

The decays of the $\tau$ lepton have intensively been studied both experimentally and theoretically. Theoretical methods range from VMD to chiral perturbation theory, see, e.g., [16 20] and references therein. The main aim of this section is to extract some MD parameters that will be used in Secs. $\mathbb{I V}$ and $\mathrm{V}$ in the calculations of decay rates of pseudoscalar mesons and cross sections of reactions involving them. Nevertheless, in order to assess the possibilities and limitations of the MD approach we consider it useful to show its predictions for the $\tau$ lepton decay modes, even if many of them have already been obtained by other authors. In some cases the MD works well, even for such a complex decay mode as $\tau^{-} \rightarrow \pi^{-} \pi^{0} \eta \nu_{\tau}$.

Some formulas presented here were derived by Tsai [21] for decays of heavy leptons before the $\tau$ lepton was actually discovered [22]. His theoretical input included lepton universality, conserved vector current (CVC) hypothesis [23], VMD in electromagnetic interactions, and the Weinberg [24] and Das-Mathur-Okubo [25] sum rules.

The formula for the partial decay width of $\tau^{-}$into a pseudoscalar meson and neutrino (see Fig. (1) is well known and can be found, e.g., in [21,26,27]. For the reader's convenience and later reference it is shown also here. 


$$
\Gamma_{\tau^{-} \rightarrow P^{-} \nu_{\tau}}=\frac{G_{F}^{2} m_{\tau^{-}}^{3} Z_{P}}{16 \pi}\left(1-\frac{m_{P}^{2}}{m_{\tau^{-}}^{2}}\right)^{2} .
$$

Using the values of $Z_{P}$ parameters as given in Table $\mathbb{1 1}$ and the mean lifetime of the $\tau^{-}$we get the branching fractions of the $\pi^{-}$and $K^{-}$mode shown in Table III together with results of the evaluation of other $\tau^{-}$decay modes described below.

The Feynman diagram that corresponds to the decay of the $\tau$ lepton to a $\rho$ meson and a neutrino is shown in Fig. 1. Using the MD Lagrangian (2.15) it is easy to write down the corresponding matrix element. The resulting formula for the partial decay width, first derived by Tsai [21], is

$$
\Gamma_{\tau^{-} \rightarrow \nu_{\tau} \rho^{-}}=\left(\frac{G_{F}\left|V_{u d}\right|}{g_{\rho}}\right)^{2} \frac{m_{\rho}^{2}}{8 \pi m_{\tau}^{3}}\left(m_{\tau}^{2}-m_{\rho}^{2}\right)^{2}\left(m_{\tau}^{2}+2 m_{\rho}^{2}\right) .
$$

Numerically, Eq. (3.2) yields a branching fraction of $19.0 \%$.

To correct for the finite $\rho$ width, we will consider the three-body decay $\tau^{-} \rightarrow \pi^{-} \pi^{0} \nu_{\tau}$ going via $\rho^{-}$in intermediate state, see Fig. 2. Let us recall first that if the decaying particle possesses spin zero or if we average over its spin states, then the usual formula for the three-body decay $a \rightarrow 1+2+3$ (see, e.g., [7], p. 176) simplifies to

$$
d \Gamma=\frac{1}{8(2 \pi)^{4} m_{a}^{2}} \overline{|\mathcal{M}|^{2}}\left|\mathbf{p}_{1}\right|\left|\mathbf{p}_{2}^{*}\right| d M_{23} d \Omega_{2}^{*}
$$

where $\mathbf{p}_{1}$ is the momentum of particle 1 in the rest frame of the decaying particle, $\mathbf{p}_{2}^{*}$ is the momentum of particle 2 in the rest frame of 2 and $3, d \Omega_{2}^{*}$ is the corresponding solid angle element, and $M_{23}$ is the mass of the 2-3 subsystem. The bar over the matrix element squared signifies the sum over the final and average over the initial states.

The interaction among a vector field and two pseudoscalar fields is described by the Lagrangian

$$
\mathcal{L}_{V P_{1} P_{2}}=i g_{V P_{1} P_{2}} V_{\mu} \varphi_{1} \overleftrightarrow{\partial^{\mu}} \varphi_{2}+\text { h.c. }
$$

If the decay $V \rightarrow P_{1}+P_{2}$ is kinematically allowed then its rate comes out from (3.4) as

$$
\Gamma_{V \rightarrow P_{1}+P_{2}}=\frac{g_{V P_{1} P_{2}}^{2}}{48 \pi m_{V}^{5}} \lambda^{3 / 2}\left(m_{V}^{2}, m_{P_{1}}^{2}, m_{P_{2}}^{2}\right)
$$

where

$$
\lambda(x, y, z)=x^{2}+y^{2}+z^{2}-2 x y-2 x z-2 y z
$$

is the so called triangle function. In the $\rho \pi \pi$ case the coupling constants for all three charge combinations have the same absolute value $g_{\rho}$.

Using Lagrangians (2.15) and (3.4), the three-body decay formula (3.3) and neglecting the difference between the $\pi^{-}$and $\pi^{0}$ masses we arrive at the partial width per unit interval in the $\pi^{-} \pi^{0}$ system mass 


$$
\frac{d \Gamma_{\tau^{-} \rightarrow \pi^{-} \pi^{0} \nu_{\tau}}}{d M}=\frac{\left(G_{F}\left|V_{u d}\right|\right)^{2}}{192 \pi^{3} m_{\tau}^{3} s}\left(m_{\tau}^{2}-s\right)^{2}\left(m_{\tau}^{2}+2 s\right)\left(s-4 m_{\pi}^{2}\right)^{3 / 2}|F(s)|^{2},
$$

where $s=M^{2}$ and

$$
|F(s)|^{2}=\frac{m_{\rho}^{4}}{\left(s-m_{\rho}^{2}\right)^{2}+m_{\rho}^{2} \Gamma_{\rho}^{2}}
$$

Let us note that $g_{\rho}$ coming from the Lagrangian (2.15) canceled with that from the $\rho^{-} \pi^{-} \pi^{0}$ vertex. To account for contributions from higher $\rho$ resonances, in actual calculations we replaced (3.8) by the $\pi$ form factor taken from [14. The final result after the integration over the allowed range of $M$ and translation into the branching fraction is $(24.4 \pm 0.4) \%$. Our value is a little bigger than that of Kühn and Santamaria 28], who used the same formula but a different form factor, but still smaller than the experimental value of $(25.24 \pm 0.16) \%$.

After consulting Fig. 2 we can see that the differential partial width of the decay $\tau^{-} \rightarrow K^{-} K^{0} \nu_{\tau}$ can be obtained from (3.7) by substituting $m_{\pi} \rightarrow m_{K}$ and multiplying by $\left(g_{\rho^{-} K^{-} K^{0}} / g_{\rho}\right)^{2}$. The latter quantity could only be obtained from the analysis of the kaon electromagnetic form factor. Here we determine its product with $\left|V_{u d}\right|^{2}$ from the experimental branching fraction. This product, denoted as $X_{\bar{K}^{0} K^{-} \rho^{+}}$, will be used as an input parameter in Sec. IV.

To get formulas for the $\tau^{-}$decay rates into $K^{*-}, a_{1}^{-}$, or $K_{1}^{-}$mesons in narrow width approximation, we only need to change the masses in (3.2) and replace $\left|V_{u d}\right|$ by $w_{K^{*}}\left|V_{u s}\right|$, $w_{a_{1}}\left|V_{u d}\right|$, or $w_{K_{1}}\left|V_{u s}\right|$, respectively.

Tsai [21] assumed that the second Weinberg sum rule [24 is saturated by narrow-width $\rho$ and $a_{1}$ mesons and got the prediction for the $\tau^{-} \rightarrow a_{1}^{-}+\nu_{\tau}$ decay rate. In our notation this situation would correspond to $w_{a_{1}}=1$. Here we treat $w_{a_{1}}$ as a phenomenological parameter and determine its value from the experimental branching fraction. The results is $w_{a_{1}}=0.8044 \pm 0.0023$. The corresponding value of the parameter $Y_{a_{1}}$, defined by Eq. (2.16) is shown in Tab. 四.

In the case of $\tau^{-} \rightarrow K_{1}^{-} \nu_{\tau}$ we proceed similarly and obtain $w_{K_{1}}=0.84 \pm 0.21$.

The value $\left(w_{K^{*}}\left|V_{u s}\right|\right)^{2}=(4.20 \pm 0.09) \times 10^{-2}$ will be determined in Sec. IVA from the experimental branching fraction of $K^{+} \rightarrow \pi^{0} e^{+} \nu_{e}$ and the full width of $K^{*+}$. The corresponding $\tau^{-} \rightarrow K^{*-} \nu_{\tau}$ branching fraction, which can be considered a prediction of the MD approach, is shown in Tab. III.

\section{A. Decays of the type $\tau^{-} \rightarrow V+P+\nu_{\tau}$}

When dealing with decay $\tau^{-} \rightarrow \omega \pi^{-} \nu_{\tau}$, see Fig. 3, we have to exploit also the Lagrangian of interaction among two vector fields and a pseudoscalar one

$$
\mathcal{L}_{V_{1} V_{2} P}=g_{V_{1} V_{2} P} \epsilon_{\mu \nu \rho \sigma} \partial^{\mu} V_{1}^{\nu} \partial^{\rho} V_{2}^{\sigma} \varphi_{P}
$$

The differential decay width in masses of the $\omega \pi^{-}$system comes out as 


$$
\begin{aligned}
\frac{d \Gamma_{\tau^{-} \rightarrow \omega \pi^{-} \nu_{\tau}}}{d M}= & \frac{\left(G_{F}\left|V_{u d}\right|\right)^{2}}{6\left(4 \pi m_{\tau}\right)^{3}}\left(\frac{g_{\rho^{-} \omega \pi^{-}}}{g_{\rho}}\right)^{2} \\
& \times \frac{1}{M^{3}}\left(m_{\tau}^{2}-M^{2}\right)^{2}\left(m_{\tau}^{2}+2 M^{2}\right) \lambda^{3 / 2}\left(M^{2}, m_{\omega}^{2}, m_{\pi}^{2}\right)\left|F\left(M^{2}\right)\right|^{2}
\end{aligned}
$$

The $\rho^{-} \omega \pi^{-}$coupling constant can be fixed using the VMD and experimental branching fraction of the radiative decay $\omega \rightarrow \gamma \pi^{0}$, as discussed in Appendix A. It was shown by Decker [29] that a simple form factor like (3.8) did not lead to a proper description of the $\omega \pi^{-}$mass spectrum [30 and that a higher $\rho$ pole had to be included. He used what was known at that time as $\rho(1600)$. Later it became clear that the $1600 \mathrm{MeV}$ region actually contains two $\rho$-like resonances. Castro and López [31] showed that a better description of the same data [30] is provided by combining $\rho(770)$ with $\rho(1450)$ rather than with $\rho(1700)$. Having fixed the admixture parameter, they obtained the branching fraction that we included in our Tab. III together with the recently published experimental value [32].

Formula (3.10) gives, after obvious modifications, also the differential decay width of not yet observed decay mode $\tau^{-} \rightarrow \phi \pi^{-} \nu_{\tau}$. Here, the $\rho^{-} \phi \pi^{-}$coupling constant can be determined in a more direct way, namely, by exploring the experimental branching fraction of $\phi \rightarrow \rho \pi$ as shown in Appendix A. Instead of performing our own analysis we again quote the result of Castro and López, who assumed that the form factor is the same as in the $\omega \pi^{-}$ case.

The calculation of the branching fraction of the decay $\tau^{-} \rightarrow K^{* 0} K^{-} \nu_{\tau}$ is complicated by the fact that two Feynman diagrams, one with $\rho^{-}$, the other with $a_{1}^{-}$in the intermediate state, see Fig. \&, contribute to the transition amplitude. The contribution of the former is proportional to the $\rho^{-} K^{* 0} K^{-}$coupling constant, the value of which can be determined by analyzing the $K^{* 0}$ and $K^{*+}$ radiative decays by means of the VMD in electromagnetic interactions. This analysis offers two solutions for $\left(g_{\rho^{-}} K^{* 0} K^{-} / g_{\rho}\right)^{2}$ that are compatible with experimental data on the $K^{*}$ radiative decays, namely, $(2.22 \pm 0.18) \mathrm{GeV}^{-2}$ and $(9.2 \pm 5.8) \times$ $10^{-2} \mathrm{GeV}^{-2}$,

If we forget for a moment about the axial current diagram and calculate the branching fraction only from the diagram with $\rho^{-}$in the intermediate state, we find that it plays a negligible role. Even for the larger solution shown above the resulting branching fraction is very small, $(6.0 \pm 0.5) \times 10^{-5}$, far below the experimental value of $(2.0 \pm 0.6) \times 10^{-3}$. It shows that the dominant contribution is provided by the diagram with $a_{1}^{-}$in the intermediate state. [n Unfortunately, we do not have any possibility to fix the $a_{1}^{-} K^{* 0} K^{-}$coupling constant. So instead of an honest calculation let us make a crude estimate of what the experimental information on the $\tau^{-} \rightarrow K^{* 0} K^{-} \nu_{\tau}$ and $\tau^{-} \rightarrow a_{1}^{-} \nu_{\tau}$ would imply if the former mode were a subprocess of the latter. Dividing their branching fractions leads to $B\left(a_{1}^{-} \rightarrow K^{* 0} K^{-}\right) \approx 1 \%$. This value does not seem to be excluded by the "possibly seen" status of this mode in [7].

Another example of the $\tau^{-}$decay modes with one pseudoscalar and one vector meson in final state is $\tau^{-} \rightarrow \rho^{-} \eta \nu_{\tau}$. This mode was considered a possible test of the Wess-Zumino term [33] for chiral anomalies [34]. The expected branching fraction lay in the interval

\footnotetext{
${ }^{3}$ We must say that this conclusion disagrees with that reached in Ref. 20.
} 
$(3.4,3.9) \times 10^{-4}$ [35]. In the MD approach we describe it by means of the hadronic vertex connecting two $\rho$ 's with the $\eta$, see Fig. . 0 introduced in Eq. 3.9. Because the narrow width approximation is not as justified as well as it was in the case of the $\omega$ and $\phi$ mesons, we complete the diagram with two pions originating from the $\rho$ and evaluate the $\tau^{-} \rightarrow \pi^{-} \pi^{0} \eta \nu_{\tau}$ branching fraction. Everything greatly simplifies if we assume that the mass difference between $\pi^{-}$and $\pi^{0}$ can be neglected. The Feynman diagram depicted in Fig. 5 then leads to

$$
\begin{aligned}
\Gamma_{\tau^{-} \rightarrow \pi^{-} \pi^{0} \eta \nu_{\tau}}= & \frac{\left(G_{F} g_{\rho \rho \eta}\left|V_{u d}\right|\right)^{2}}{9(4 \pi)^{5} m_{\tau}^{3} m_{\rho}^{4}} \int_{2 m_{\pi}}^{m_{\tau}-m_{\eta}} d M_{2}\left(M_{2}^{2}-4 m_{\pi}^{2}\right)^{3 / 2}\left|F\left(M_{2}^{2}\right)\right|^{2} \\
& \times \int_{M_{2}+m_{\eta}}^{m_{\tau}} \frac{d M_{1}}{M_{1}^{3}}\left(m_{\tau}^{2}-M_{1}^{2}\right)^{2}\left(m_{\tau}^{2}+2 M_{1}^{2}\right) \lambda^{3 / 2}\left(M_{1}^{2}, M_{2}^{2}, m_{\eta}^{2}\right)\left|F\left(M_{1}^{2}\right)\right|^{2} .
\end{aligned}
$$

One yet unknown parameter is the coupling constant in the $\rho \rho \eta$ vertex. As shown in Ap-

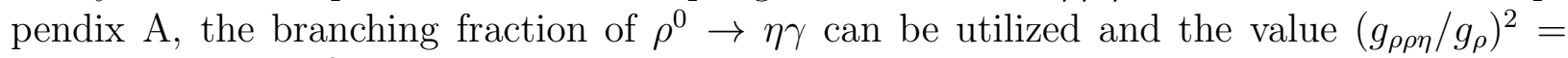
$(15.1 \pm 2.8) \mathrm{GeV}^{-2}$ is obtained. Using the form factor [14] and integrating Eq. (3.11) numerically we end up with the branching fraction $(1.79 \pm 0.33) \times 10^{-3}$, which agrees perfectly with the experimental value of $(1.71 \pm 0.28) \times 10^{-3}$.

\section{B. Decay $\tau^{-} \rightarrow \pi^{-} \eta \nu_{\tau}$}

The experimental upper limit for the branching fraction of the $\tau^{-} \rightarrow \pi^{-} \eta \nu_{\tau}$ mode $(1.4 \times$ $10^{-4}$ ) indicates that this mode is suppressed relative to $\tau^{-} \rightarrow \rho^{-} \eta \nu_{\tau}$, which we considered above, by at least one order of magnitude in spite of the larger phase space available. This can easily be understood within the MD approach. In fact, if the spin-parity conservation laws are strictly enforced in conjunction with those based on isospin invariance, then there is no pseudoscalar, vector, or axial-vector meson that can couple to the $\pi^{-} \eta$ system. The only possibility to realize the transition from $W^{-}$to this system is via the $a_{0}^{-}$scalar meson, see Fig. 6. It gives us a chance to gain some information about the strength of the $a_{0}^{-} W^{-}$ interaction, as shown in the following.

The dominant decay mode of the $a_{0}^{-}$meson is $a_{0}^{-} \rightarrow \pi^{-} \eta$. It allows us to replace the decay $\tau^{-} \rightarrow \pi^{-} \eta \nu_{\tau}$ in our considerations by a simpler one, namely, $\tau^{-} \rightarrow a_{0}^{-} \nu_{\tau}$. . $^{\text {The }}$ coupling of scalar mesons to the gauge bosons is similar to that of pseudoscalar mesons, as shown by a comparison of (2.18) and (2.22). We can therefore use Eq. (3.1) to find an upper bound on the scalar-meson decay constant of the $a_{0}^{-}$meson. The result is $f_{a_{0}^{-}}<7 \mathrm{MeV}$. For comparison, the pseudoscalar decay constant of the $\pi^{-}$meson is about $131 \mathrm{MeV}$.

\footnotetext{
${ }^{4}$ This decay was proposed as a clear test for the existence of second-class vector current by Leroy and Pestieau [36] soon after the discovery of the $\tau$ lepton [22].
} 


\section{PROCESSES CONTAINING THE $P-P-V^{+}$VERTEX WITH $V^{+}$COUPLED TO $W^{+}$}

In this section we will consider charged weak current decays of pseudoscalar mesons $\left(P_{1}\right)$ into a pseudoscalar meson $\left(P_{2}\right)$ and an additional system, which may be an $\ell \nu$ pair, another pseudoscalar meson, a vector meson, or an axial-vector meson. According to the MD hypothesis the processes of this kind proceed by coupling the pseudoscalar meson pair to a charged vector meson $(V)$, which in turn couples to a charged gauge boson. The latter finally converts into one of the systems mentioned above. The $P_{1} \rightarrow P_{2}+V$ transition is governed by the Lagrangian (3.4). It is useful to introduce the quantity

$$
X_{P_{1} P_{2} V}=Y_{V}\left(\frac{g_{V P_{1} P_{2}}}{g_{\rho}}\right)^{2}
$$

which will enter all our formulas for decay rates in this section. Parameter $Y_{V}$ is defined by Eq. (2.16), $g_{V P_{1} P_{2}}$ is the coupling constant in the Lagrangian (3.4).

Our general strategy will be to determine the quantities (4.1) from some of the experimentally known branching fractions of semileptonic decays and then use them for making predictions for other decay modes. A notable exception is the decay $\pi^{+} \rightarrow \pi^{0} e^{+} \nu_{e}$, which proceeds via the $\rho^{+}$meson. Here, the quantity under consideration is simply given by the $u d$ element of the CKM matrix, $X_{\pi^{+} \pi^{0} \rho^{+}}=\left|V_{u d}\right|^{2}$, and is thus well known.

\section{A. Decays of the type $P_{1} \rightarrow P_{2}+\ell^{+} \nu_{\ell}$}

The generic Feynman diagram for the weak decay of a pseudoscalar meson $P_{1}$ into another pseudoscalar meson $P_{2}$ and an $\ell^{+} \nu_{\ell}$ pair is shown in Fig. 17. The corresponding matrix element can easily be written on the basis of the lepton part of the standard model Lagrangian and Eqs. (2.15) and (3.4).

$$
\mathcal{M}=G_{F} w_{V} V_{V} \frac{g_{V P_{1} P_{2}}}{g_{\rho}} \frac{m_{V}^{2}}{m_{V}^{2}-t}\left[\left(p_{1}+p_{2}\right)^{\mu}-\frac{m_{P_{1}}^{2}-m_{P_{2}}^{2}}{m_{V}^{2}}\left(p_{1}-p_{2}\right)^{\mu}\right] \bar{l} \gamma_{\mu}\left(1-\gamma_{5}\right) \nu,
$$

where $p_{1}\left(p_{2}\right)$ is the four-momentum of the incoming (outgoing) meson and $t=\left(p_{1}-p_{2}\right)^{2}$ is the square of the four-momentum transfer from $P_{1}$ to $P_{2}$. Obviously, $t$ is also equal to the

mass squared of the $\ell^{+} \nu_{\ell}$ system. The evaluation based on Eqs. (3.3) and (4.2) gives, after the integration over the lepton momentum direction in the $\ell^{+} \nu_{\ell}$ rest frame, the following formula for the differential partial width:

$$
\frac{d \Gamma_{P_{1} \rightarrow P_{2} \ell^{+} \nu_{\ell}}}{d t}=\frac{G_{F}^{2} X_{P_{1} P_{2} V}}{3\left(4 \pi m_{P_{1}}\right)^{3}} \frac{t-m_{\ell}^{2}}{t^{3}} \lambda^{1 / 2}\left(m_{P_{1}}^{2}, m_{P_{2}}^{2}, t\right)\left[\varphi_{1}(t)-\varphi_{2}(t)\right]\left(\frac{m_{V}^{2}}{m_{V}^{2}-t}\right)^{2},
$$

where

$$
\begin{aligned}
\varphi_{1}(t)= & 2 t^{4}-(4 x+4 y+z) t^{3}+\left[2(x-y)^{2}+z(2 x+2 y-z)\right] t^{2} \\
& +2 z\left[(x-y)^{2}+z(x+y)\right] t-4 z^{2}(x-y)^{2}, \\
\varphi_{2}(t)= & \frac{3 t z}{r^{2}}(2 r-t)(t-z)(x-y)^{2} .
\end{aligned}
$$


We used the notation $r=m_{V}^{2}, x=m_{P_{1}}^{2}, y=m_{P_{2}}^{2}$, and $z=m_{\ell}^{2}$. Integrating Eq. (4.3) in case of the $\pi^{+} \rightarrow \pi^{0} e^{+} \nu_{e}$ decay (usually referred to as $\pi_{e 3}$ ), we get a branching fraction of $(1.0041 \pm 0.0021) \times 10^{-8}$. The error comes from the mean $\pi^{ \pm}$lifetime we used to convert the decay rate into the branching ratio and from the $V_{u d}$ element of the CKM matrix. The agreement between our result and the experimental value of $(1.025 \pm 0.034) \times 10^{-8}$ is perfect.

As it has already been mentioned, the $\pi_{e 3}$ decay is exceptional because the coupling constant in the hadronic vertex just cancels with the $g_{\rho}$ coming from the MD Lagrangian (2.15) and the $w_{\rho^{+}}$parameter is exactly one, as it follows from the normalization of the pion electromagnetic form factor. In other semileptonic decays the formulas for branching fractions contain badly known or unknown parameters $X_{P_{1} P_{2} V}$ defined in Eq. (4.1). Some experimental branching fractions have been explored to determine those parameters, with resulting values shown in Table $[\mathrm{IV}$. Others, shown in Tab. $\mathrm{V}$, provide the check of the soundness of the MD results.

For example, the branching fraction of the decay $K^{+} \rightarrow \pi^{0} e^{+} \nu_{e}$ is used to fix the value of $X_{K^{+} \pi^{0} K^{*+}}$ at $(1.206 \pm 0.015) \times 10^{-2}$; those of $K^{+} \rightarrow \pi^{0} \mu^{+} \nu_{\mu}, K_{L}^{0} \rightarrow \pi^{ \pm} e^{\mp} \bar{\nu}_{e}\left(\nu_{e}\right)$, and $K_{L}^{0} \rightarrow \pi^{ \pm} \mu^{\mp} \bar{\nu}_{\mu}\left(\nu_{\mu}\right)$ then come as predictions of the MD. The result for electron mode of $K_{L}^{0}$ is somewhat higher than the experimental value. It may signal the presence of isospin symmetry violating effects [37,38]. After taking the experimental value of $\left|V_{u s}\right|$ and determining the coupling constant ratio $g_{K^{*+} K^{+} \pi^{0}}^{2} / g_{\rho}^{2}=0.2872 \pm 0.0051$ from the $\rho$ and $K^{*+}$ decay widths we isolate $w_{K^{*+}}=0.929 \pm 0.013$. The deviation of the latter from unity is what one would expect for an $S U_{f}(3)$ breaking effect.

For other decay modes such a detailed analysis cannot be performed because the hadronic coupling constants of vector meson resonances are either inaccessible for fundamental reasons (e.g., hadronic decay is not kinematically allowed) or because the decay widths are poorly known. We are thus left with the $X_{P_{1} P_{2} V}$ values shown in Tab. IV], without the possibility to extract the $w_{V}$ parameters. But it does not hamper our ability to predict the branching fractions of related processes $P_{1} \rightarrow P_{2}+P_{3}$ and $P_{1} \rightarrow P_{2}+V(A)$.

A very interesting situation is in the semileptonic decays of $B$-mesons. Frequent decay modes $B^{0} \rightarrow D^{-} \ell^{+} \nu_{\ell}, B^{+} \rightarrow \bar{D}^{0} \ell^{+} \nu_{\ell}$, and $B_{s}^{0} \rightarrow D_{s}^{-} \ell^{+} \nu_{\ell}$ cannot be explained within the MD framework without assuming the existence of a vector meson with both charm and beauty, $B_{c}^{*+}$. But such a meson has not yet been discovered experimentally. In order to proceed further we simply assume that it does exist and choose its mass at $6.34 \mathrm{GeV} / c^{2}$, as determined by Godfrey and Isgur [39] in a relativized quark model with chromodynamics. This value agrees with results of other potential models [40]. We will return to this question, which is of vital importance for the MD hypothesis, in Sec. VII.

In Tab. $\nabla$ we also show the predictions for semileptonic decay modes with the $\tau$ lepton. It is natural to ask to which extent the branching ratio of the $\tau$ and light lepton modes can discriminate among various models. Let us mention that for the $B^{+} \rightarrow \pi^{-}$semileptonic decays our ratio is 0.52 , while the recent estimate by Khodjamirian and Rückl 41] is 0.7-0.8.

The branching fractions are not the only outcome of the MD approach that can be com-

\footnotetext{
${ }^{5}$ The issue of applicability the MD approach to decays of heavy mesons will be addressed in Sec. IVD.
} 
pared with experimental data. Any experimentally observable quantity can be calculated. Let us consider as an example the form factors $f_{+}(t)$ and $f_{-}(t)$ of the $K_{e 3}^{+}$decay, which are defined by

$$
\mathcal{M}=\frac{G_{F} V_{u s}}{\sqrt{2}}\left[f_{+}(t)\left(p_{1}+p_{2}\right)^{\mu}+f_{-}(t)\left(p_{1}-p_{2}\right)^{\mu}\right] \bar{\ell} \gamma_{\mu}\left(1-\gamma_{5}\right) \nu
$$

Differential decay rate for arbitrary form factors is shown in Appendix B. If we now compare Eq. 4.2 with the definition (4.5) of $f_{+}(t)$, we see that in the MD approach the $t$-dependence of the latter is given by

$$
f_{+}(t)=f_{+}(0) \frac{m_{K^{*+}}^{2}}{m_{K^{*+}}^{2}-t} .
$$

The comparison with the linear parametrization used by experimentalists is shown in Fig. 8. A faster than linear rise of the MD form factor may explain why the experimentally determined slope parameters in the $K_{\mu 3}$ decays are higher than those in $K_{e 3}$.

Furthermore, from (4.2) we are getting the form factor ratio

$$
\frac{f_{-}(t)}{f_{+}(t)}=-\frac{m_{K^{+}}^{2}-m_{\pi^{0}}^{2}}{m_{K^{*+}}^{2}}=-0.28367 \pm 0.00016,
$$

which should be compared to the experimental value of $-0.35 \pm 0.15$. Equations (4.6) and (4.7) were first derived by Dennery and Primakoff [42], who saturated the dispersion relations for the form factors by a $K^{*}$ pole.g

\section{B. Decays of the type $P_{1} \rightarrow P_{2}+P_{3}$}

The generic Feynman diagram of the processes we are going to consider now is shown in Fig. 9. The parent pseudoscalar meson $P_{1}$ undergoes the strong interaction conversion into $P_{2}$, one of the outgoing pseudoscalar mesons, and a charged (virtual) vector meson $V$. The latter couples according to (2.15) to the $W$ boson, which in turn converts to the second outgoing pseudoscalar meson $P_{3}$. To simplify the discussion we will consider only positively charged $V$ 's, which means neutral or positively charged $P_{1}$ 's and, correspondingly, negatively charged or neutral $P_{2}$ 's. This convention clearly shows which of the two final-state pseudoscalar mesons is coupled to the gauge boson. Of course, we automatically handle also the charge conjugate modes.

The mechanism considered here does not operate in all $P_{1} \rightarrow P_{2}+P_{3}$ transitions. It cannot explain any of the decays into two neutral mesons. Also some charged modes cannot run in this way. Let us take as an example $D_{s}^{+} \rightarrow \bar{K}^{0} K^{+}$. There does not exist any vector meson that would appear together with $\bar{K}^{0}$ as a result of the strong conversion of $D_{s}^{+}$. And what accompanies $K^{+}$in such a conversion is $D^{* 0}$, which cannot couple to any of the weak gauge bosons.

\footnotetext{
${ }^{6}$ In fact, they considered two vector resonances. One of them, $K^{*}(730)$, was abandoned later on.
} 
The partial decay width comes out from the Feynman diagram in Fig. 9 as

$$
\Gamma_{P_{1} \rightarrow P_{2}+P_{3}}=\frac{G_{F}^{2}}{16 \pi m_{1}^{3}} X_{P_{1} P_{2} V} Z_{P_{3}}\left(m_{P_{1}}^{2}-m_{P_{2}}^{2}\right)^{2} \lambda^{1 / 2}\left(m_{P_{1}}^{2}, m_{P_{2}}^{2}, m_{P_{3}}^{2}\right) .
$$

Parameters $X_{P_{1} P_{2} V}$, defined by Eq. (4.1), have already been assigned numerical values using experimental information on some semileptonic decay modes, as shown in Table IV. Similarly, the parameters $Z_{P_{3}}$ are defined by Eq. (2.20). Their values were determined from the leptonic branching fractions of pseudoscalar mesons and are shown in Table [1].

The results obtained from (4.8) for various input and output mesons were converted to branching fractions by means of experimental lifetimes. They can be divided into three groups. In Tab. $\nabla]$ we present calculated branching fractions that agree with experimental data. Their less lucky companions are listed in Tab. VII. We defer the discussion about possible meaning of discrepancies between our results and empirical values to Sec. VII. The last group, shown in Tab. VIII, comprises the branching fractions that have not been measured yet. When the experimental information becomes more complete some of the modes listed there may fall into the first category, some into the second one.

\section{Decays of the type $P_{1} \rightarrow P_{2}+V(A)$}

Keeping in mind our convention about charges of the parent pseudoscalar mesons, the flavor-changing decays we are going to analyze now can proceed in the lowest order of the MD approach only through the diagram depicted in Fig. 10. Because the vector $(V)$ and axial-vector $(A)$ mesons couple to the charged gauge bosons in the same way, we can study the two modes, one with an outgoing vector meson, the other with an outgoing axial-vector meson, simultaneously. We will label either of those two mesons as $M$, freeing the index $V$ for the intermediate vector meson that connects the hadronic vertex with $W^{+}$. The partial decay width summed over the spin projections of $M$ reads

$$
\Gamma_{P_{1} \rightarrow P_{2}+M}=\frac{G_{F}^{2} m_{M}^{2} X_{P_{1} P_{2} V} Y_{M}}{8 \pi g_{\rho}^{2} m_{P_{1}}^{3}}\left(\frac{m_{V}^{2}}{m_{V}^{2}-m_{M}^{2}}\right)^{2} \lambda^{3 / 2}\left(m_{P_{1}}^{2}, m_{P_{2}}^{2}, m_{M}^{2}\right) .
$$

The branching fractions calculated from (4.9) and mean life times of the parent mesons $\left(D^{+}, D^{0}, D_{s}^{+}, B^{+}\right.$, and $\left.B^{0}\right)$ are shown in Tables $\mathbb{\mathbb { X }}$ and $\mathbb{X}$ together with experimental values. The former table lists the decay modes for which the MD results do not contradict the experiment. As most of the empirical values are given only as upper bounds at present, some modes may move in future to Table $\mathbf{X}$, which contain the MD results that disagree with the data.

\section{Meson dominance and decays of heavy mesons}

The decays rates of heavy mesons containing a heavy quark and a light antiquark, or vice versa, are usually calculated using the heavy quark effective theory [43]. The careful reader has probably noticed when inspecting Tables $\mathrm{V} \mathbb{X}$ that we used the MD formulas to calculate also the decay fractions of the heavy mesons $D, D_{s}, B$, and $B_{s}$. It seems to go 
against the spirit of the MD, as it has been declared in Sec. I1, because the energies in the parent rest frame of the outgoing particles are large. But, as we will argue, the use of MD for calculating the branching ratios of different light meson modes is well justified.

In fact, what matters is the virtuality (four-momentum squared) flowing through the junction where a meson and the weak gauge boson meet. Let's speak, for definiteness, about the decay of $B^{0}$ into $D^{-}$and a light meson $M^{+}$(where now $M$ can be $P, V$, or $A$ ), which is supposedly dominated by the $B_{c}^{*+}$. See Figs. 9 and 10. Because the meson $M^{+}$is on the mass shell, the virtuality in the $W^{+} M^{+}$junction is equal to the mass of $M$ squared, i.e., it is same as in the definitions of the weak effective Lagrangians in Sec. II. The story at the opposite end of the $W^{+}$line, in the $B_{c}^{*+} W^{+}$junction, is different. Here, the virtuality is far from the $B_{c}^{*+}$ mass squared, and the coupling parameter may be different from the $w_{B_{c}^{*+}}$, which is defined for the on-shell $B_{c}^{*+}$. Similar changes may occur in the strong $B^{0} D^{-} B_{c}^{*+}$ vertex, where again the dependence on the virtualities of participating mesons cannot be ruled out. The absolute predictions of the decay rates may thus be unreliable. But we cannot make the absolute predictions anyhow because we know neither $w_{B_{c}^{*+}}$ nor $g_{B^{0} D^{-} B_{c}^{*+}}$, which combine into $X_{B^{0} D^{-} B_{c}^{*+}}$, see Eqs. (2.16) and (4.1). We can only calculate the ratios of decay rates for different mesons $M^{+}$. When these mesons are light, the differences among the virtualities in the $W^{+} B_{c}^{*+}$ junction will be small in comparison with the mass of the $B_{c}^{*+}$ meson. The virtuality modified coefficients $X_{B^{0} D^{-} B_{c}^{*+}}$ will have approximately the same value and will simply cancel out when a ratio of the decay rates is calculated. The same happens when a light meson mode is compared to the $\ell^{+} \nu_{\ell}$ one.

To push things to the edge we calculated the branching ratios of various decay modes of the $B_{c}$ meson, containing both heavy valence quark and antiquark. This meson has not been discovered yet, but reliable calculations of its mass and other properties exist in the literature, see, e.g., [39.40]. Also the prospects of its impending discovery are bright, see [14] and references therein. Following Godfrey and Isgur [39] we used in our calculations the $B_{c}$ mass of $6.27 \mathrm{GeV} / c^{2}$. The results of the MD approach are compared to the predictions of some existing more fundamental models in Table X].

\section{E. Meson-electron induced binary reactions}

Let us consider the following weak-interaction binary reactions of projectile mesons incident on target electrons: $\pi^{+} e^{-} \rightarrow \pi^{0} \nu_{e}, K^{+} e^{-} \rightarrow \pi^{0} \nu_{e}, \pi^{+} e^{-} \rightarrow \bar{K}^{0} \nu_{e}$, and $K^{+} e^{-} \rightarrow K^{0} \nu_{e}$. First two of them are exoenergetic, whereas the laboratory kinetic energy thresholds for the remaining two are $223.1 \mathrm{GeV}$ and $3.381 \mathrm{GeV}$, respectively. As a consequence of the special kinematics (electron as a target), the reactions remain in the low center-of-mass energy range $\left(s,|t|<1 \mathrm{GeV}^{2}\right)$ even for the highest meson beam energies available. With view toward successful description of the semileptonic decays of pion and kaons, we believe that the MD approach is suitable also for calculating the cross sections of the low-energy reactions that are related to those decays by crossing symmetry. The corresponding Feynman diagram is depicted in Fig. 11. To make the differential cross section formula concise, we introduced $x=m_{P_{1}}^{2}, y=m_{e}^{2}, z=m_{P_{2}}^{2}$, and $r=m_{V}^{2}$. The formula then reads

$$
\frac{d \sigma_{P_{1} e \rightarrow P_{2} \nu}}{d t}=\frac{G_{F}^{2} X_{P_{1} P_{2} V}(\hbar c)^{2}}{8 \pi \lambda(s, x, y)}\left(\frac{r}{r-t}\right)^{2}\left[\phi_{1}(s, t)+\frac{x-z}{r^{2}} \phi_{2}(s, t)\right]
$$


with

$$
\begin{aligned}
& \phi_{1}(s, t)=4 s(s-x-y-z)+4 x z+y^{2}+(4 s-y) t \\
& \phi_{2}(s, t)=2 r y(2 s+t-y-2 z)+y(x-z)(y-t) .
\end{aligned}
$$

Total cross section as a function of the kinetic energy of incident meson for the four mesonelectron reactions mentioned above were obtained by numerical integration and are shown in Fig. 12.

\section{F. Antineutrino-electron induced mesonic reactions}

The electron antineutrino energies required for meson-pair production off target electrons are very high. We show four most favorable final states with the threshold antineutrino energies in parentheses: $\pi^{-} \pi^{0}(73.8 \mathrm{GeV}), K^{-} \pi^{0}(387 \mathrm{GeV}), \bar{K}^{0} \pi^{-}(398 \mathrm{GeV})$, and $K^{-} K^{0}$ $(962 \mathrm{GeV})$. Also the fact that the electron antineutrinos are less copious than the muon ones by a factor of $10^{-4}$ in the high energy antineutrino beams, produced by the $\pi_{\ell 2}^{-}$decays, makes the experimental observation of this kind of reaction tricky. On the other hand, as the cms energy of the two-meson system remains small, the transverse momenta of outgoing mesons will also be small. The reaction products will thus be concentrated in a very narrow cone in the laboratory system polar angle with relatively small energy spread. This, and also the negative total charge, may help to identify this kind of reactions. The evaluation of the total cross section that corresponds to the Feynman diagram in Fig. 13 gives the result

$$
\begin{aligned}
\sigma_{\bar{\nu}_{e}+e^{-} \rightarrow P_{1}+P_{2}}= & \frac{G_{F}^{2}(\hbar c)^{2} X_{P_{1} P_{2} V}}{24 \pi r^{2} s^{3}(s-y)^{2}}|F(s)|^{2} \lambda^{1 / 2}(s, x, z) \\
& \times\left[2 r^{2} s^{3} \lambda(s, x, z)+2 r^{2} y^{2}(2 y-3 s)(x-z)^{2}+r^{2} s y\left(3 s^{2}-y^{2}\right)\right. \\
& \left.\times(2 x+2 z-s)+3 s y(x-z)^{2}(s-y)^{2}(s-2 r)\right],
\end{aligned}
$$

where $x=m_{P_{1}}^{2}, y=m_{e}^{2}, z=m_{P_{2}}^{2}, r=m_{V}^{2}$, and

$$
|F(s)|^{2}=\frac{m_{V}^{4}}{\left(s-m_{V}^{2}\right)^{2}+m_{V}^{2} \Gamma_{V}^{2}} .
$$

For channels with the $\rho$ resonance in the $s$-channel we replaced function (4.12) by the form factor taken from Ref. [14, in which the experimental data on $e^{+} e^{-} \rightarrow \pi^{+} \pi^{-}$were fit with a formula exhibiting the correct analytic behaviour. In this way we have accounted for a possible contribution from higher $\rho$ recurrences. For reactions with a $\pi K$ system in the final state, which go through the $K^{*}$ resonance in the $s$-channel, we do not have such a possibility. The single-pole formula (4.12) with energy dependent $K^{*}$ width was used. The dependence of the total cross section on the incident antineutrino energy for all four final states is shown in Fig. 14. 


\section{MESON DOMINANCE AND NEUTRAL FLAVOR CHANGING DECAY MODES}

The processes we are going to deal with now are usually classified [0] as flavor changing $(\Delta S=1, \Delta C=1)$ weak neutral current decay modes. This label is a little misleading for some of them, e.g., $K^{+} \rightarrow \pi^{+} \ell^{+} \ell^{-}$. In the calculations based on the standard model the latter is described in terms of diagrams that almost all contain 455 the charged gauge boson $W^{ \pm}$, i.e., the charged weak current. Also in the MD approach we will calculate branching fractions of this and similar decay modes using the diagrams where charged mesons are attached to the charged weak gauge bosons. Only in a part of this class of processes $\left(K^{+} \rightarrow \pi^{+} \bar{\nu} \nu\right.$, for example) the genuine weak neutral current operates in conjunction with the charged one, which can only change the flavor.

We start with considering the decay mode $K^{+} \rightarrow \pi^{+} \ell^{+} \ell^{-}$, which was investigated theoretically already before its discovery in 1975 [46]. References to this early period can be found in [45]. Later works include [47 49] and references therein. Present theoretical understanding of this decay in the framework of chiral perturbation theory has recently been summarized in [50,51]. The theoretical prediction based on [47] contains one unknown parameter. When extracting it from the experimental branching fraction of the dilectron mode, a two-fold ambiguity remained. It was resolved by choosing the solution that fit the $e^{+} e^{-}$ mass spectrum [52] better. Then the prediction for the $\mu^{+} \mu^{-}$mode can be made.

In the MD approach we will describe the decay $K^{+} \rightarrow \pi^{+} \ell^{+} \ell^{-}$by the diagram sketched in Fig. 15, forgetting for a while about other possible diagrams. In order to evaluate the corresponding decay rate we assume that the interaction among the $a_{1}, \rho$, and $\pi$ mesons is governed by the Lagrangian density

$$
\mathcal{L}_{a_{1} \rho \pi}=i g_{a_{1} \rho \pi} \sum_{i, j, k} C_{i ; j, k} V_{j \alpha \beta}^{\dagger} \partial^{\alpha} \varphi_{k}^{\dagger} A_{i}^{\beta}
$$

with

$$
V_{j \alpha \beta}=\partial_{\alpha} V_{j \beta}-\partial_{\beta} V_{j \alpha}
$$

being the vector field strength tensor. Symbols $A_{i}, V_{j}$, and $\varphi_{k}$ denote the field operators for $a_{1}, \rho$, and $\pi$ mesons, respectively. Italic indices label various charge states of a particular meson and $C_{i ; j, k}$ is the $\mathrm{SU}(2)$ Clebsch-Gordan coefficient. The $a_{1} \rightarrow \rho \pi$ decay width comes from (5.1) as

$$
\Gamma_{a_{1} \rightarrow \rho+\pi}=\frac{g_{a_{1} \rho \pi}^{2}}{96 \pi m_{a_{1}}^{3}} \lambda^{1 / 2}(x, y, z)\left[(x-y-z)^{2}+\frac{y}{2 x}(x-y+z)^{2}\right],
$$

where $x=m_{a_{1}}^{2}, y=m_{\rho}^{2}$, and $z=m_{\pi}^{2}$. Substituting the experimental value of $\Gamma_{a_{1}} \approx 400 \mathrm{MeV}$ into Eq. (5.3) we get $g_{a_{1} \rho \pi}^{2} \approx 260 \mathrm{GeV}^{-2}$.

A straightforward evaluation of the diagram depicted in Fig. 15 leads to the following formula for the differential width in the $\ell^{+} \ell^{-}$mass $M$

$$
\begin{aligned}
\frac{d \Gamma_{K^{+} \rightarrow \pi^{+} \ell^{+} \ell^{-}}}{d M}= & \frac{\left(G_{F} g_{a_{1} \rho \pi} \alpha\right)^{2}}{48 \pi g_{\rho}^{4} m_{K^{+}}^{3}} Y_{a_{1}^{+}} Z_{K^{+}} \\
& \times \lambda^{3 / 2}\left(m_{K^{+}}^{2}, m_{\pi^{+}}^{2}, M^{2}\right) \sqrt{M^{2}-4 m_{\ell}^{2}}\left(1+\frac{2 m_{\ell}^{2}}{M^{2}}\right)\left(\frac{m_{\rho}^{2}}{m_{\rho}^{2}-M^{2}}\right)^{2} .
\end{aligned}
$$


Besides $g_{a_{1} \rho \pi}$ there are two other nontrivial parameters entering formula (5.4), $Y_{a_{1}^{+}}$and $Z_{K^{+}}$. Their values can be found in Tables [I and II], respectively. When we integrate (5.4) over the full range of dielectron masses and use the experimental value of the $K^{+}$lifetime, we get the branching fraction $B\left(K^{+} \rightarrow \pi^{+} e^{+} e^{-}\right) \approx 3.1 \times 10^{-7}$. The experimental value is $(2.74 \pm 0.23) \times 10^{-7}$. The uncertainty of our result comes from the $a_{1} \rho \pi$ coupling constant, which is given by the poorly known (and understood) width of the $a_{1}$ meson. But this uncertainty disappears when we calculate the branching ratio of the $\mu^{+} \mu^{-}$and $e^{+} e^{-}$modes, which is a function only of the masses of participating particles. In Table XII we show therefore the branching fraction of the $K^{+} \rightarrow \pi^{+} \mu^{+} \mu^{-}$mode normalized by the experimental value of the dielectron one.

As we have already indicated, there are other MD diagrams that can contribute to the amplitude of $K^{+} \rightarrow \pi^{+} \ell^{+} \ell^{-}$decay mode. First of all, it might be a diagram obtained from that in Fig. 15 by substitutions $a_{1}^{+} \rightarrow \rho^{+}$and $\rho^{0} \rightarrow \omega, \phi$. But it vanishes identically as a consequence of the presence of the totally antisymmetric Levi-Civitta tensor in the hadronic vertex together with the pion momentum in the $W^{+} \pi^{+}$junction.

Then we have diagrams that contain only pseudoscalar mesons. They are generated by taking the basic diagram in which $K^{+}$converts to $\pi^{+}$via $W^{+}$and attaching a virtual photon alternatively to all possible lines. It can be shown that the sum of those diagrams is vanishing. To simplify the discussion we will show it here in the limit of an infinitely heavy $W$ boson. In this limit we can introduce the following effective Lagrangian for the weak interaction between pions and kaons:

$$
\mathcal{L}_{\pi K}=-\frac{G_{F}}{\sqrt{2}} f_{\pi} V_{u d} f_{K} V_{u s} \partial^{\mu} \varphi_{\pi} \partial_{\mu} \varphi_{K}^{\dagger}+\text { h.c. . }
$$

After switching on the electromagnetic interaction by the minimal substitution principle, we are getting not only the usual terms describing the emission of a photon from the pion and kaon lines, but also contact terms generated from (5.5). The one-photon part of the electromagnetic interaction Lagrangian thus reads

$$
\mathcal{L}_{\gamma}=i e a_{\mu}\left[\varphi_{\pi}^{\dagger} \partial^{\mu} \varphi_{\pi}+\varphi_{K}^{\dagger} \partial^{\mu} \varphi_{K}-\frac{G_{F}}{\sqrt{2}} f_{\pi} V_{u d} f_{K} V_{u s}\left(\varphi_{\pi}^{\dagger} \partial^{\mu} \varphi_{K}+\varphi_{K}^{\dagger} \partial^{\mu} \varphi_{\pi}\right)\right],
$$

where $a_{\mu}$ denotes the electromagnetic field operator. Now it is easy to check that the matrix element for the photon production (both real or virtual) calculated as the sum of the emission from kaon line, emission from pion line, and the contact term, see Fig. 16, is identically zero. It is a consequence of our treating pions and kaons as elementary quanta. In 455 this kind of contribution was calculated assuming nontrivial electromagnetic structure of the participating mesons. The result is proportional to the difference between kaon and pion electromagnetic radii squared.

The last two diagrams conceivable in the lowest order of MD are illustrated in Fig. 17. The matrix element with the $K^{*+}$ in the intermediate state vanishes identically. The contribution from $K_{1}^{+}$is nonvanishing but small. This can be seen from the following: When we consider this part of the transition amplitude separately, ignoring the contribution from the $a_{1}$ diagram (Fig. 5.4), the resulting branching fraction of $K^{+} \rightarrow \pi^{+} e^{+} e^{-}$can be expressed in terms of the decay width of $K_{1}^{+} \rightarrow K^{+} \gamma$. To get the correct experimental number for the former, the latter had to be unrealistically high, about $40 \%$. 
To complete our discussion about the $K^{+} \rightarrow \pi^{+} e^{+} e^{-}$mode let us stress that in the MD approach we have gotten a parameter-free description of its decay rate, dominated by the $a_{1}$ diagram in Fig. 15. Other meson diagrams give smaller contributions. Nevertheless, they will have to be taken into account when a more detailed comparison with the next generation of more precise data is made. Our result suggests that in any approach based on the standard model it is important to consider the diagram depicted in Fig. 18a. It represents a seed for the class of diagrams, like the one shown in Fig. 18b, into which it develops after QCD corrections are included. This class corresponds to the most important meson diagram, Fig. [15.

Finally, it has to be stressed that the successful description of the decay $K^{+} \rightarrow \pi^{+} e^{+} e^{-}$ was possible because the short-distance part of the amplitude, which contains contributions from the electromagnetic penguin $s \rightarrow d+\gamma^{*}$, the $Z^{0}$ penguin $s \rightarrow d+Z^{0 *}$, and the $W$ box diagram, is about three orders of magnitude smaller than the long-distance part [6].

To get an estimate of the branching fraction of the transition of charmed pseudoscalar mesons $D^{+}$and $D_{s}^{+}$to a dilepton and a pion we will again use Eq. (5.4) with obvious modifications. The results shown in Table XTI should really be considered as an order-ofmagnitude estimate because (i) the $a_{1}$ in Fig. 5.4 is very far from its mass-shell and its coupling to $W$ may differ from that assumed in (2.15); (ii) also higher charged pseudoscalar [for example, $\pi(1300)]$ or tensor $\left[a_{2}(1320)\right]$ resonances that couple to the $\rho \pi, \omega \pi$, or $\phi \pi$ system can appear in the intermediate state. But it is highly improbable that the observed branching fractions will be dramatically lower than those shown in Table XII as a result of destructive interference. In fact, the matrix element now is not a mere number, but a function of the dilepton mass and the angle between the dilepton and pion. A substantial cancellation would require the same functional dependence of different contributions.

In spite of all the crudeness of our estimates we can say that the MD approach predicts the branching fraction of $D^{+} \rightarrow \pi^{+} \ell^{+} \ell^{-}$that is at least by an order of magnitude higher than the prediction of a standard model calculation [53]. But even the MD prediction is about two orders of magnitude below the present-day experimental limit. The same is true for the MD prediction in the $D_{s} \rightarrow \pi^{+} \ell^{+} \ell^{-}$case.

To check the applicability of the MD approach to neutral current processes mediated by the neutral gauge boson $Z$ we calculated the long-distance contribution to the $\mathrm{CP}$ conserving decay $K^{+} \rightarrow \pi^{+} \bar{\nu} \nu$. In the MD approach it proceeds mainly according to diagram displayed in Fig. 19. Its differential partial width is given by

$$
\frac{d \Gamma_{K^{+} \rightarrow \pi^{+} \bar{\nu} \nu}}{d t}=\frac{G_{F}^{4} g_{a_{1} \rho \pi}^{2} Y_{a_{1}^{+}} Z_{K^{+}}}{3 g_{\rho}^{4}\left(8 \pi m_{K^{+}}\right)^{3}}\left(1-2 \sin ^{2} \theta_{W}\right)^{2} t^{2} \lambda^{3 / 2}\left(m_{K^{+}}^{2}, m_{\pi^{+}}^{2}, t\right)\left(\frac{m_{\rho}^{2}}{m_{\rho}^{2}-t}\right)^{2},
$$

where $t=\left(p_{K}-p_{\pi}\right)^{2}$ is the four-momentum transfer squared, or, equivalently, the mass of the $\bar{\nu} \nu$ system. The integrated branching fraction $(7.9 \pm 0.6) \times 10^{-18}$ does not have any observational value. From the theoretical point of view it is interesting and perhaps surprising that our value is practically equal to the recent estimate $7.71 \times 10^{-18}$ (error not given) [54] obtained from the finite part of the one-loop amplitude in the chiral perturbation theory. 


\section{RELATION BETWEEN THE CONSERVED VECTOR CURRENT AND MESON DOMINANCE HYPOTHESES}

The conserved vector current (CVC) hypothesis is a useful concept in the weak interaction phenomenology. From a pragmatic point of view it enables the decay rate of some flavor conserving weak processes to be related to the data on hadron production in $e^{+} e^{-}$ annihilation 26,21,55,56. It is therefore natural to ask what is the relation between MD and CVC, which of the two approaches is more general, and which has more predictive power.

It is evident that MD can be applied also to processes during which the flavor of the hadronic system changes, whereas the CVC cannot be. Let us therefore consider only the flavor conserving processes with the weak vector current. At first sight it seems that MD in weak interactions is a straightforward consequence of CVC hypothesis and $\rho^{0}$ dominance in electromagnetic interactions. If it were true, the two concepts would lead to the same results in the region where their domains of validity overlap.

A typical process of this type is the $\pi_{e 3}$ decay $\pi^{+} \rightarrow \pi^{0} e^{+} \nu_{e}$. We have shown that one can get good agreement with the data by calculating its branching fraction from the MD Lagrangian (2.15) without any further assumption. It has also been claimed for a long time (see, e.g., 26,27]) that the agreement of the CVC result with experiment is perfect and lends strongest support to the CVC hypothesis. To examine this assertion let us sketch briefly the central point of the CVC derivation.

From general principles it follows that the matrix element for the $\pi_{e 3}$ decay has the form (4.5). The continuity equation for the conserved weak vector current requires

$$
\left(p_{1}-p_{2}\right)_{\mu}\left[\left(p_{1}+p_{2}\right)^{\mu} f_{+}(t)+\left(p_{1}-p_{2}\right)^{\mu} f_{-}(t)\right]=0
$$

what leads to the relation

$$
f_{-}(t)=-\frac{m_{\pi^{+}}^{2}-m_{\pi^{0}}^{2}}{t} f_{+}(t)
$$

Because $t$ is the mass squared of the $\ell \nu$ system, it cannot vanish. In the limit of exact isospin symmetry we have $m_{\pi^{+}}=m_{\pi_{0}}$ and therefore

$$
f_{-}(t) \equiv 0
$$

The usual CVC result for the $\pi_{e 3}$ branching fraction is obtained by assuming that the identity (6.3) holds also when isospin symmetry is broken. The other assumptions state that the function $f_{+}(t)$ can be replaced by a constant in the small $t$ range allowed kinematically in the $\pi_{e 3}$ decay and that the relations among different components of the electroweak isovector vector current remain same as in the case of exact isospin symmetry. The final formula can be found in Ref. [27. .7 Although different from the MD formula, after numerical evaluation it gives practically identical value, which agrees with the experimental branching ratio very well (see Table $\nabla$ ).

\footnotetext{
${ }^{7}$ In Ref. [26] additional approximations were made, which lowered the result by $2.5 \sigma$. Equation (7.15) in [27] contains an obvious misprint: $\pi^{5}$ should be read as $\pi^{3}$.
} 
But let us look at the CVC procedure described above a little more closely. The assumption that relation (6.3) is valid also when isospin symmetry is broken violates the relation (6.1). So the usual CVC result is, in fact, obtained not by assuming the conservation of the vector current, but rather assuming a special type of its nonconservation, namely such that results in Eq. (6.3).

If we strictly enforce the conservation of the vector current by honoring Eq. (6.2), which follows from it, we obtain the $\pi_{e 3}$ branching fraction of $(0.8872 \pm 0.0019) \times 10^{-8}$, which disagrees with the contemporary experimental value $(1.025 \pm 0.034) \times 10^{-8}$.

To conclude: We found a process for which the CVC hypothesis and MD give different results. Meson dominance in the flavor conserving vector current sector thus represents a dynamic assumption that is different from what would be obtained by merging the CVC hypothesis with the VMD in electromagnetic interactions. The case of the $\pi_{e 3}$ decay suggests that MD is better suited for description of the processes in which the isospin invariance is broken.

\section{CONCLUSIONS AND COMMENTS}

The hypothesis that the weak interaction of hadronic systems at low energies is dominated by the coupling of the vector, axial-vector, and pseudoscalar mesons to the gauge bosons has been scrutinized. The strength of the weak coupling of the $\rho(770)$ meson is uniquely determined by vector-meson dominance in electromagnetic interactions; flavor and chiral symmetry breaking effects modify the coupling of other vector mesons and axial-vector mesons. Corresponding strengths parameter and their products with (mostly unknown) strong interaction coupling constants constitute the free parameters of our approach. They are fixed by experimental data on the branching fractions of the selected decay modes of the $\tau$ lepton and semileptonic decay modes of pseudoscalar mesons. Some hadronic coupling constants were determined from the widths of strong and radiative decays.

After fixing the parameters, many decay rates of the $\tau$ lepton and pseudoscalar mesons $\left(\pi, K, D, D_{s}, B\right.$, and $\left.B_{s}\right)$ have been calculated and compared to experimental data. They fall into three categories:

- Decay modes where the calculated result is in good agreement with observation. One can expect that these modes, when calculated in the framework of the standard model, are dominated by the weak quark-antiquark annihilation and creation diagrams. The nicest example in this category is the semileptonic decay $\pi^{+} \rightarrow \pi^{0} e^{+} \nu_{e}$. The calculated branching fraction is $(1.0041 \pm 0.0021) \times 10^{-8}$, while experiment says $(1.025 \pm 0.034) \times$ $10^{-8}$. Many nonleptonic decays are also well described. For example, $D^{+} \rightarrow \pi^{0} \pi^{+}$, $D_{s}^{+} \rightarrow \eta \pi^{+}, B^{+} \rightarrow \bar{D}^{0} D_{s}^{+}, B^{0} \rightarrow D^{-} \rho^{+}$. Also the branching fraction of a quite complex mode $\tau^{-} \rightarrow \eta \pi^{-} \pi^{0} \nu_{\tau}$ agrees nicely with the experimental figure. So does that of the "neutral current flavor changing" mode $K^{+} \rightarrow \pi^{+} e^{+} e^{-}$.

\footnotetext{
${ }^{8}$ For the differential decay rate formula see Appendix B.
} 
- Decay modes where the calculation disagrees with experimental data. Here the standard model diagrams that do not have an analogy in the meson dominance approach ( $W$ emission or absorption from a quark line, penguin diagrams, box diagrams, etc.) are expected to dominate. The two pion decays of the $K$ mesons are a typical example.

- Decay modes that have not been measured yet. Some meson-dominance predictions: $B\left[K_{L}^{0} \rightarrow K^{ \pm} e^{\mp} \bar{\nu}_{e}\left(\nu_{e}\right)\right]=(3.4 \pm 0.6) \times 10^{-9}, B\left(B^{0} \rightarrow \pi^{-} \tau^{+} \nu_{\tau}\right)=(9.4 \pm 3.1) \times 10^{-5}$, $B\left(B_{s}^{0} \rightarrow D_{s}^{-} \tau^{+} \nu_{\tau}\right)=(1.9 \pm 0.6) \%, B\left(D_{s}^{+} \rightarrow \eta^{\prime} K^{+}\right)=(1.3 \pm 0.5) \times 10^{-3}, B\left(K^{+} \rightarrow\right.$ $\left.\pi^{+} \mu^{+} \mu^{-}\right)=(6.2 \pm 0.5) \times 10^{-8}$.

An upper limit on the presence of the second-class vector current was obtained using the experimental limit on the $\tau^{-} \rightarrow \pi^{-} \eta \nu_{\tau}$ branching fraction. In terms of the scalar decay constant of the $a_{0}^{-}$meson it reads $f_{a_{0}^{-}}<7 \mathrm{MeV}$. The upper bound is about twenty times smaller that the decay constant of the $\pi^{+}$meson.

What comes as a surprise is the ability of the MD approach to provide a parameterfree description of the flavor changing $\Delta Q=0$ process $K^{+} \rightarrow \pi^{+} e^{+} e^{-}$. Also the MD tree diagram calculation of the long-distance part of the flavor changing neutral current decay $K^{+} \rightarrow \pi^{+} \nu \bar{\nu}$ gives the same result as a one-loop evaluation in the chiral perturbation theory with certain prescription for handling the divergent part.

The cross sections of several not yet observed reactions of pions, kaons, and electron antineutrinos with target electrons come as predictions of the meson dominance approach. These include, e.g., $\pi^{+} e^{-} \rightarrow \pi^{0} \nu_{e}, K^{+} e^{-} \rightarrow \pi^{0} \nu_{e}, \bar{\nu}_{e} e^{-} \rightarrow \pi^{-} \pi^{0}$.

The transitions $B \rightarrow \bar{D}$ and $B_{s} \rightarrow D_{s}^{-}$where the final state meson is accompanied by an $\ell^{+} \nu_{\ell}$ system, or a positively charged pseudoscalar, vector, or axial-vector meson cannot be explained within the MD approach without assuming the existence of the as yet unobserved vector meson $B_{c}^{*+}$. The results of MD calculations depend on its mass. We used the value obtained from the potential models [39,40]. The question arises whether it would not be possible to determine the $B_{c}^{*+}$ mass from the experimental branching ratios of various decay modes using the MD formulas. In order to answer this question we increased the $B_{c}^{*+}$ mass by $0.5 \mathrm{GeV}$ and recalculated the branching fractions. The biggest decrease was experienced by semileptonic decay modes. But even here it was only by $3.3 \%$. It makes any effort to predict the $B_{c}^{*+}$ mass using the MD approach unrealistic. Prospects of producing the $B_{c}^{*+}$ mesons were assessed already in early papers, e.g., [39.40]. The present state of art can be found in [44] and in references therein. There is a hope that an observable number of $B_{c}$ and $B_{c}^{*}$ events can be produced at LEP and at Tevatron.

On the theoretical side, the relation between the meson dominance and the conserved vector current hypothesis has been clarified.

\section{ACKNOWLEDGMENTS}

I am indebted to P.J. Ellis, J.I. Kapusta, A.Z. Dubničková, M. Mojžiš, and J. Pišút for stimulating discussions, to J.A. Thompson for information about the E865 experimental program at AGS and for discussions about the physics at $\phi$ factories. Critical remarks by S. Rudaz and A. Vainshtein during the seminar in Minneapolis in September 1995 and those of V. Černý (Bratislava, August 1996) forced me to formulate the basic assumptions 
and virtues of this approach more clearly. This work was initiated during my stay at the University of Minnesota, which was supported by the U.S. Department of Energy under Contract No. DOE/DE-FG02-87ER-40328 and completed at the Department of Energy's Institute for Nuclear Theory at the University of Washington. The partial support provided by the latter is gratefully acknowledged. In Bratislava, the work was a part of the project VEGA 1/1323/96.

\section{APPENDIX A: DETERMINING THE $V V P$ COUPLING CONSTANTS FROM DATA ON STRONG AND RADIATIVE DECAYS}

If the decay $V_{1} \rightarrow V_{2}+P$ is energetically allowed, the $V_{1} V_{2} P$ coupling constant can be determined from its empirical decay rate. Using Lagrangian (3.9) we easily derive the formula

$$
\Gamma_{V_{1} \rightarrow V_{2}+P}=\frac{g_{V_{1} V_{2} P}^{2}}{96 \pi m_{V_{1}}^{3}} \lambda^{3 / 2}\left(m_{V_{1}}^{2}, m_{V_{2}}^{2}, m_{P}^{2}\right) .
$$

Triangle function $\lambda$ is defined by Eq. (3.6). The experimentally given branching fraction of $\phi \rightarrow \rho \pi$ includes three different final states, which would be equally probable if the isospin symmetry was exact. If we assume that the latter is violated only through mass differences, we obtain $g_{\phi \rho^{+} \pi^{-}}^{2}=(1.17 \pm 0.07) \mathrm{GeV}^{-2}$.

To get the expression for the rate of radiative decay $V_{1} \rightarrow \gamma+P$ we only need to replace $V_{2}$ by $\gamma$.

$$
\Gamma_{V_{1} \rightarrow \gamma+P}=\frac{g_{V_{1} \gamma P}^{2}}{96 \pi m_{V_{1}}^{3}}\left(m_{V_{1}}^{2}-m_{P}^{2}\right)^{3} .
$$

VMD in electromagnetic interactions enables to express the $g_{V_{1} \gamma P}$ coupling constant by means of the hadronic ones. For radiative decay $\omega \rightarrow \gamma \pi^{0}$ the situation is simple because only $\rho^{0}$ can couple to the $\omega \pi^{0}$ system. Using (1.1) we can write

$$
g_{\omega \gamma \pi^{0}}^{2}=\frac{4 \pi \alpha}{g_{\rho}^{2}} g_{\omega \rho^{0} \pi^{0}}^{2}
$$

and calculate

$$
\left(\frac{g_{\omega \rho^{0} \pi^{0}}}{g_{\rho}}\right)^{2}=\frac{24 m_{\omega}^{3}}{\alpha\left(m_{\omega}^{2}-m_{\pi^{0}}^{2}\right)^{3}} \Gamma_{\omega \rightarrow \gamma \pi^{0}}=(5.40 \pm 0.32) \mathrm{GeV}^{-2}
$$

The radiative decay $\rho^{0} \rightarrow \eta \gamma$ can proceed only via the strong $\rho^{0} \eta \rho^{0}$ vertex because the isospin conservation prevents coupling of the $\rho^{0} \eta$ system to either $\omega$ or $\phi$. Another consequence of the isoscalar character of the $\eta$ meson is that the quantity

$$
\left(\frac{g_{\rho \eta \rho}}{g_{\rho}}\right)^{2}=\frac{24 m_{\rho}^{3}}{\alpha\left(m_{\rho}^{2}-m_{\eta}^{2}\right)^{3}} \Gamma_{\rho^{0} \rightarrow \eta \gamma}=(15.1 \pm 2.8) \mathrm{GeV}^{-2}
$$

has the same value for all charge states of $\rho$. 
The case of $K^{*}$ radiative decays is most complicated because the resulting amplitude is given as a coherent sum of three amplitudes with $\gamma$ coupled to $\rho, \omega$, and $\phi$. In spite of this complication we can determine the $K^{*} K \rho$ coupling constants because the $\omega$ and $\phi$ contributions to $K^{*+} \rightarrow K^{+} \gamma$ are equal to those to $K^{* 0} \rightarrow K^{0} \gamma$, whereas the $\rho^{0}$ contribution changes sign. We are thus getting the set of equations

$$
\begin{aligned}
(x+y)^{2} & =a_{+} \\
(-x+y)^{2} & =a_{0},
\end{aligned}
$$

where $x=g_{K^{*+} K^{+} \rho^{0}} / g_{\rho}, y$ stands for the expression that contains only isoscalar coupling constants, and

$$
a_{c}=\frac{24 m_{K^{* c}}^{3}}{\alpha\left(m_{K^{* c}}^{2}-m_{K^{c}}^{2}\right)^{3}} \Gamma_{K^{* c} \rightarrow K^{c}+\gamma}
$$

for $c=+, 0$, Using the relation $g_{K^{*+} K^{0} \rho^{+}}^{2}=2 g_{K^{*+} K^{+} \rho^{0}}^{2}$, which follows from isospin invariance, we eventually get two values of $\left(g_{K^{*+} K^{0} \rho^{+}} / g_{\rho}\right)^{2}$ that are compatible with experimental data on radiative $K^{*}$ decays, namely $2.21 \pm 0.14 \mathrm{GeV}^{-2}$ and $(9.2 \pm 2.9) \times 10^{-2} \mathrm{GeV}^{-2}$.

\section{APPENDIX B: DECAY $P_{1} \rightarrow P_{2}+\ell^{+} \nu_{\ell}$ : GENERAL FORM FACTORS}

The general form of the matrix element is

$$
\mathcal{M}=C\left[f_{+}(t)\left(p_{1}+p_{2}\right)^{\mu}+f_{-}(t)\left(p_{1}-p_{2}\right)^{\mu}\right] \bar{\ell} \gamma_{\mu}\left(1-\gamma_{5}\right) \nu,
$$

where $p_{1}\left(p_{2}\right)$ is the four-momentum of the incoming (outgoing) pseudoscalar meson and $t=\left(p_{1}-p_{2}\right)^{2}$ is the mass squared of the lepton system. Using (3.3) and integrating over the solid angle in the $\ell^{+} \nu_{\ell}$ rest frame we get the following expression for the differential decay rate:

$$
\begin{aligned}
\frac{d \Gamma_{P_{1} \rightarrow P_{2} \ell^{+} \nu_{\ell}}}{d t}= & \frac{|C|^{2}}{3\left(4 \pi m_{P_{1}}\right)^{3}} \frac{t-m_{\ell}^{2}}{t^{3}} \lambda^{1 / 2}\left(m_{P_{1}}^{2}, m_{P_{2}}^{2}, t\right)\left\{\varphi_{1}(t)\left|f_{+}(t)\right|^{2}\right. \\
& \left.+6 z t(x-y)(t-z) \operatorname{Re}\left[f_{+}^{*}(t) f_{-}(t)\right]+3 z t^{2}(t-z)\left|f_{-}(t)\right|^{2}\right\} .
\end{aligned}
$$

Function $\varphi_{1}(t)$ is defined in Eq. (4.4). Also the meaning of other symbols is same as in Sec. IVA: $x=m_{P_{1}}^{2}, y=m_{P_{2}}^{2}, z=m_{\ell}^{2}$.

To get the total rate of $\pi^{+} \rightarrow \pi^{0} e^{+} \nu_{e}$ that follows from the requirement of exact conservation of the vector current, see Section VI, we need to integrate (B1) with $C=G_{F} V_{u d}$, $f_{+}(t)=1$, and $f_{-}(t)=-(x-y) / t$. 


\section{REFERENCES}

[1] Y. Nambu, Phys. Rev. 106, 1366 (1957); W.R. Frazer and J.R. Fulco, Phys. Rev. Lett. 2, 365 (1959); J.J. Sakurai, Ann. Phys. (N.Y.) 11 (1960) 1; M. Gell-Mann and F. Zachariasen, Phys. Rev. 124, 953 (1961); M. Gell-Mann, Phys. Rev. 125, 1067 (1962); Y. Nambu and J.J. Sakurai, Phys. Rev. Lett. 8, 79 (1962); 8, 191(E) (1962); M. Gell-Mann, D. Sharp and W. Wagner, Phys. Rev. Lett. 8, 261 (1962).

[2] S.L. Glashow, Nucl. Phys. 22, 579 (1961); S. Weinberg, Phys. Rev. Lett. 19, 1264 (1967); A. Salam in Proc. 8th Nobel Symposium, Aspenäsgården, 1968, ed. N. Svartholm (Almquist and Wiksell, Stockholm, 1968) p. 367.

[3] S.L. Glashow, J. Iliopoulos, and L. Maiani, Phys. Rev. D 2, 1258 (1970).

[4] L.-M. Chounet, J.-M. Gaillard, and M.K. Gaillard, Phys. Rep. C 4, 199 (1972).

[5] D.Yu. Bardin and E.A. Ivanov, Fiz. Elem. Chastits At. Yadra 7, 286 (1978) [Sov. J. Part. Nucl. 7, 286 (1978)].

[6] G. Buchalla, A.J. Buras, and M.E. Lautenbacher, Rev. Mod. Phys. 68, 1125 (1996).

[7] R.M. Barnett et al. (Particle Data Group), Phys. Rev. D 54, 1 (1996).

[8] D. Scora and W. Isgur, Phys. Rev. D 52, 2783 (1995).

[9] D. Du, G. Lu, and Y. Yang, Phys. Lett. B 387, 187 (1996).

[10] N. Cabibbo, Phys. Rev. Lett. 10, 531 (1963).

[11] M. Kobayashi and T. Maskawa, Prog. Theor. Phys. 49, 652 (1973).

[12] S. Weinberg, Phys. Rev. 112, 1375 (1958).

[13] L. Beldjoudi, Tran N.Truong, Phys. Lett. B 344, 419 (1995).

[14] M.E. Biagini, S. Dubnička, E. Etim, and P. Kolář, Nuovo Cimento A 104, 363 (1991).

[15] C.W. Bernard, J.N. Labrenz, and A. Soni, Phys. Rev. D 49, 2536 (1994).

[16] R. Decker, M. Finkemeier, P. Heiliger, and H.H Jonsson, Z. Phys. C 70, 247 (1996).

[17] M. Finkemeier and E. Mirkes, Z. Phys. C 69, 243 (1996).

[18] H. Davoudias and M.B. Wise, Phys. Rev. D 53, 2523 (1996).

[19] G. Colangelo, M. Finkemeier, and R. Urech, Phys. Rev. D 54, 4403 (1996).

[20] B.A. Li, Phys. Rev. D (to be published) (hep-ph/9606402).

[21] Y.S. Tsai, Phys. Rev. D 4, 2821 (1971);

[22] M.L. Perl et al., Phys. Rev. Lett. 35, 1489 (1975).

[23] S.S. Gerstein and J.B. Zeldovich, Zh. Eksp. Teor. Fiz. 29, 698 (1955) [Sov. Phys.-JETP 2, 576 (1956)]; R.P. Feynman and M. Gell-Mann, Phys. Rev. 109, 193 (1958).

[24] S. Weinberg, Phys. Rev. Lett. 18, 507 (1967).

[25] T. Das, V.S. Mathur, and S. Okubo, Phys. Rev. Lett. 18, 761 (1967).

[26] L.B. Okun, Leptons and Quarks (North-Holland Pub. Co., Amsterdam, New York, 1982); Leptony i kvarki (Nauka, Moskva 1981).

[27] H. Pietschmann, Weak Interactions-Formulae, Results and Derivations (Springer Verlag, Wien, New York, 1983), p. 174.

[28] J.H. Kühn and A. Santamaria, Z. Phys. C 48, 445 (1990).

[29] R. Decker, Z. Phys. C 36, 487 (1987).

[30] H. Albrecht et al., Phys. Lett. B 185, 223 (1987).

[31] G. López Castro and D.A. López Falcón, Phys. Rev. D 54, 4400 (1996).

[32] ALEPH Collaboration, D. Buskulic et al., Z. Phys. C 70, 579 (1996).

[33] J. Wess and B. Zumino, Phys. Lett. B 37, 95 (1971). 
[34] R. Fischer, J. Wess, and F. Wagner, Z. Phys. C 3, 313 (1980); G. Aubrecht, N. Chahrouri, and K. Slanec, Phys. Rev. D 24, 1318 (1981).

[35] G. Kramer and W.F. Palmer, Z. Phys. C 25, 195 (1984).

[36] C. Leroy and J. Pestieau, Phys. Lett. B 72, 398 (1978).

[37] H. Leutwyler and M. Roos, Z. Phys. C 25, 91 (1984).

[38] J. Gasser and H. Leutwyler, Nucl. Phys. B 250, 517 (1985).

[39] S. Godfrey and N. Isgur, Phys. Rev. D 32, 189 (1985).

[40] E. Eichten and F. Feinberg, Phys. Rev. D 23, 2724 (1981); S.S. Gershtein et al., Yad. Fiz. 48, 515 (1988) [Sov. J. Nucl. Phys. 48, 327 (1988)].

[41] A. Khodjamirian and R. Rückl, preprint WUE-ITP-96-020, MPI-PhT/96-108 (Oct. 1996) hep-ph/9610367.

[42] P. Dennery and H. Primakoff, Phys. Rev. 131, 1334 (1963).

[43] M.B. Voloshin and M.A. Shifman, Yad. Fiz. 47, 801 (1988) [Sov. J. Nucl. Phys. 47, 511 (1988)]; N. Isgur and M.B. Wise, Phys. Lett. B 232, 113 (1989); 237, 527 (1990); E. Eichten and B. Hill, ibid. 234, 511 (1990); H. Georgi, ibid. 240, 447 (1990); F. Hussain, J.G. Körner, K. Schilcher, G. Thompson, and Y.L. Wu, ibid. 240, 447 (1990); A.F. Falk, H. Georgi, B. Grinstein, and M.B. Wise, Nucl. Phys. B 343, 1 (1990); J.G. Körner and G. Thompson, Phys. Lett. B 240, 185 (1991); M. Neubert, ibid. 264, 455 (1991); 338, 84 (1994); Phys. Rep. 245, 259 (1994).

[44] Y.-Q. Chen, OHSTPY-HEP-T-96-029 (September 1996), hep-ph/9610239; C.-H. Chang, Y.-Q. Chen, and R.J. Oakes, Phys. Rev. D 54, 4344 (1996).

[45] A.I. Vainstein, V.I. Zakharov, L.B. Okun, and M.A Shifman, Yad. Fiz. 24, 820 (1976) [Sov. J. Nucl. Phys. 24, 427 (1976)].

[46] P. Bloch et al., Phys. Lett. B 56, 201 (1975).

[47] G. Ecker, A. Pich, E. de Rafael, Nucl. Phys. B 291, 692 (1987).

[48] L. Bergström and P. Singer, Phys. Rev. D 43, 1568 (1991).

[49] S. Fajfer, Z. Phys. C 71, 307 (1996).

[50] A. Pich, Rare Kaon Decays, invited talk at the Workshop on K Physics, Orsay, France, May 30-June 4, 1996. Available as hep-ph/9610243.

[51] G. Ecker, Status of Chiral Perturbation Theory, talk given at the Workshop on Heavy Quarks at Fixed Target, St. Goar, Germany, Oct. 3-6, 1996. To appear in the Proceedings. Also available as hep-ph/9611346.

[52] C. Alliegro et al., Phys. Rev. Lett. 68, 278 (1992).

[53] J.L. Hewett, T. Takeuchi, and S. Thomas, in Electroweak Symmetry Breaking and Beyond the Standard Model, edited by T. Barklow, S. Dawson, H.E. Haber, and S. Siegrist (World Scientific, in press). Also available as hep-ph/9603391.

[54] C.Q. Geng, I.J. Hsu, and Y.C. Lin, Phys. Lett. B 355, 569 (1995).

[55] F.J. Gilman and S.H. Rhie, Phys. Rev. D 31, 1066 (1985);

[56] S.I. Eidelman and V.N. Ivanchenko, Phys. Lett. B 257, 437 (1991); Nucl. Phys. B Proc. Suppl. 40, 131 (1995).

[57] CLEO Collaboration, J.P. Alexander et al., CLNS-96-1419 (July 1996).

[58] C.-H. Chang and Y.-Q. Chen, Phys. Rev. D 49, 3399 (1994).

[59] M. Lusignoli and M. Masetti, Z. Phys. C 51, 549 (1991).

[60] D. Du and Z. Wang, Phys. Rev. D 39, 1342 (1989).

[61] S.J. Brodsky and G.P. Lepage, Phys. Rev. D 22, 2157 (1980); S.J. Brodsky and C.R. Ji, 
Phys. Rev. Lett. 55, 2257 (1985); A. Szczepaniak, E.M. Henley, and S.J. Brodsky, Phys. Lett. B 243, 287 (1990).

[62] N. Isgur, D. Scora, B. Grinstein, and M. Wise, Phys. Rev. D 39, 799 (1989).

[63] M. Wirbel, B. Stech, and M. Bauer, Z. Phys. C 29, 637 (1985). 


\section{TABLES}

TABLE I. Parameters $Y_{V}$ and $Y_{A}$, defined by Eq. (2.16) and characterizing the coupling of vector and axial-vector mesons to the charged gauge bosons, their sources, and values of parameters $w$ extracted from them.

\begin{tabular}{lccl}
\hline \hline $\mathrm{V}, \mathrm{A}$ & $Y_{V}, Y_{A}$ & $w_{V}, w_{A}$ & Source \\
\hline$\rho^{+}$ & $0.9479 \pm 0.0019$ & 1 & $\left|V_{u d}\right|^{2}$ \\
$K^{*+}$ & $(4.20 \pm 0.09) \times 10^{-2}$ & $0.929 \pm 0.013$ & $K^{+} \rightarrow \pi^{0} e^{+} \nu_{e}, K^{*} \rightarrow K \pi$ \\
$D_{s}^{*+}$ & $0.7 \pm 0.5$ & $0.83 \pm 0.33$ & $B^{0} \rightarrow D^{-} D_{s}^{*+}$ \\
$a_{1}^{+}$ & $0.6134 \pm 0.0032$ & $0.8044 \pm 0.0023$ & $\tau^{-} \rightarrow a_{1}^{-} \nu_{\tau}$ \\
$K_{1}^{+}$ & $(3.4 \pm 1.7) \times 10^{-2}$ & $0.84 \pm 0.21$ & $\tau^{-} \rightarrow K_{1}^{-} \nu_{\tau}$ \\
\hline \hline
\end{tabular}

TABLE II. Parameters $Z_{P}$ characterizing the coupling of pseudoscalar mesons to the charged gauge boson and their sources. For definition, see Eq. (2.20).

\begin{tabular}{lcl}
\hline \hline $\mathrm{P}$ & $Z_{P}\left(\mathrm{GeV}^{2}\right)$ & Source \\
\hline$\pi^{+}$ & $(1.6419 \pm 0.0010) \times 10^{-2}$ & $\pi^{+} \rightarrow \mu^{+} \nu_{\mu}+\mu^{+} \nu_{\mu} \gamma$ \\
$K^{+}$ & $(1.247 \pm 0.004) \times 10^{-3}$ & $K^{+} \rightarrow \mu^{+} \nu_{\mu}+\mu^{+} \nu_{\mu} \gamma$ \\
$D^{+}$ & $(2.2 \pm 0.5) \times 10^{-3}$ & $f_{D^{+}}$from [15], $\left|V_{c d}\right|$ from [0] \\
$D_{s}^{+}$ & $(1.1 \pm 0.5) \times 10^{-1}$ & $D_{s}^{+} \rightarrow \mu^{+} \nu_{\mu}$ \\
\hline \hline
\end{tabular}


TABLE III. Branching fractions of the $\tau$ lepton calculated in the MD approach and comparison with experimental data. Column $\mathrm{C}$ shows the meson coupled to the weak gauge boson.

\begin{tabular}{llccl}
\hline \hline Final state & $\mathrm{C}$ & Meson Dominance result & Data & Notes \\
\hline$\pi^{-} \nu_{\tau}$ & $\pi^{-}$ & $(10.91 \pm 0.06) \%$ & $(11.31 \pm 0.15) \%$ & \\
$K^{-} \nu_{\tau}$ & $K^{-}$ & $(7.13 \pm 0.04) \times 10^{-3}$ & $(7.1 \pm 0.5) \times 10^{-3}$ & \\
$\pi^{-} \pi^{0} \nu_{\tau}$ & $\rho^{-}$ & $(24.4 \pm 0.4) \%$ & $(25.24 \pm 0.16) \%$ & $(\mathrm{a})$ \\
$K^{-} K^{0} \nu_{\tau}$ & $\rho^{-}$ & $(1.22 \pm 0.56) \%$ & $(1.55 \pm 0.28) \times 10^{-3}$ & $(\mathrm{~b})$ \\
$\pi^{-} \omega \nu_{\tau}$ & $\rho^{-}$ & $(1.20 \pm 0.48) \times 10^{-5}$ & $(1.84 \pm 0.05 \pm 0.14) \%$ & $(\mathrm{c}, \mathrm{d})$ \\
$\pi^{-} \phi \nu_{\tau}$ & $\rho^{-}$ & $(1.79 \pm 0.33) \times 10^{-3}$ & $(1.71 \pm 0.28) \times 10^{-3}$ & $(\mathrm{c})$ \\
$\eta \pi^{-} \pi^{0} \nu_{\tau}$ & $\rho^{-}$ & $(1.06 \pm 0.03) \%$ & $(1.28 \pm 0.08) \%$ & $(\mathrm{a})$ \\
$K^{*}(892)^{-} \nu_{\tau}$ & $K^{*-}$ & & $(18.11 \pm 0.37) \%$ & $(\mathrm{~d}, \mathrm{e}, \mathrm{f})$ \\
$a_{1}^{-} \nu_{\tau}$ & $a_{1}^{-}$ & & $(8 \pm 4) \times 10^{-3}$ & $(\mathrm{e}, \mathrm{g})$ \\
$K_{1}(1400)^{-} \nu_{\tau}$ & $K_{1}^{-}$ & & $<1.4 \times 10^{-4}$ & $(\mathrm{~h})$ \\
$\eta \pi^{-} \nu_{\tau}$ & $a_{0}^{-}$ & & & \\
\hline \hline
\end{tabular}

(a) The normalization is determined by the VMD in QED. (b) Used to fix $X_{\rho^{-} K^{-} K^{0}}=$ $0.64 \pm 0.12$, which differs from what one would get from the $S U_{f}(4)$ coupling constant ratio by about 20\%. (c) Calculation by Castro and Falcón [31]. (d) Experimental value taken from [32]. (e) MD calculation in the narrow width approximation. (f) Used to fix $Y_{a_{1}}$. (g) Used to fix $Y_{K_{1}}$. (h) Coupling of the $\pi^{-} \eta$ system to $\pi^{-}, \rho^{-}$, or $a_{1}^{-}$is forbidden by the strong interaction and spin-parity conservation laws. This mode put a limit on the $a_{0}^{-}(980)$ decay constant $f_{a_{0}^{-}}<7 \mathrm{MeV}$.

TABLE IV. Parameters $X_{P_{1} P_{2} V}$, defined by Eq. (4.1): Numerical values and their sources.

\begin{tabular}{lllcll}
\hline \hline$P_{1}$ & $P_{2}$ & $V$ & $X_{P_{1} P_{2} V}$ & Source & Notes \\
\hline$\pi^{+}$ & $\pi^{0}$ & $\rho^{+}$ & $(0.9479 \pm 0.0020)$ & $\left|V_{u d}\right|^{2}$ \\
$K^{+}$ & $\pi^{0}$ & $K^{*+}$ & $(1.206 \pm 0.015) \times 10^{-2}$ & $K^{+} \rightarrow \pi^{0} e^{+} \nu$ & \\
$K^{0}$ & $\pi^{-}$ & $K^{*+}$ & $(2.412 \pm 0.030) \times 10^{-2}$ & $2 \times X_{K^{+} \pi^{0} K^{*+}}$ \\
$D^{+}$ & $\pi^{0}$ & $D^{*+}$ & $(8.9 \pm 3.4) \times 10^{-3}$ & $D^{+} \rightarrow \pi^{0} \ell^{+} \nu$ \\
$D^{0}$ & $\pi^{-}$ & $D^{*+}$ & $(1.8 \pm 0.7) \times 10^{-3}$ & $2 \times X_{D^{+} \pi^{0} D^{*+}}$ \\
$D^{+}$ & $\bar{K}^{0}$ & $D_{s}^{*+}$ & $0.263 \pm 0.015$ & $=X_{D^{0} K^{-} D_{s}^{*+}}$ \\
$D^{0}$ & $K^{-}$ & $D_{s}^{*+}$ & $0.263 \pm 0.015$ & $D^{0} \rightarrow K^{-} \mu^{+} \nu$ & $D_{s}^{+} \rightarrow \eta \ell^{+} \nu$ \\
$D_{s}^{+}$ & $\eta$ & $D_{s}^{*+}$ & $0.139 \pm 0.039$ & $D_{s}^{+} \rightarrow \eta^{\prime} \ell^{+} \nu$ & \\
$D_{s}^{+}$ & $\eta^{\prime}$ & $D_{s}^{*+}$ & $0.18 \pm 0.07$ & $X_{B^{+} \pi^{-} B^{*+} / 2}$ & $B^{0} \rightarrow \pi^{-} \ell^{+} \bar{\nu}_{\ell}$ \\
$B^{+}$ & $\pi^{0}$ & $B^{*+}$ & $(4.3 \pm 1.4) \times 10^{-7}$ & $B^{0} \rightarrow D^{-} \ell^{+} \nu$ & (a) \\
$B^{0}$ & $\pi^{-}$ & $B^{*+}$ & $(8.5 \pm 2.8) \times 10^{-7}$ & $=X_{B^{+} D^{-} B_{c}^{*+}}$ & (a) \\
$B^{+}$ & $\bar{D}^{0}$ & $B_{c}^{*+}$ & $(3.5 \pm 0.9) \times 10^{-4}$ & $B_{s}^{0} \rightarrow D_{s}^{-} \ell^{+} \nu$ & (a) \\
$B^{0}$ & $D^{-}$ & $B_{c}^{*+}$ & $(3.5 \pm 0.9) \times 10^{-4}$ & $\tau^{-} \rightarrow K^{-} K^{0} \nu_{\tau}$ & \\
$B_{s}^{0}$ & $D_{s}^{-}$ & $B_{c}^{*+}$ & $(1.3 \pm 0.4) \times 10^{-3}$ & & \\
$\bar{K}^{0}$ & $K^{-}$ & $\rho^{+}$ & $(0.64 \pm 0.12)$ & \\
\hline \hline
\end{tabular}

(a) Existence of $B_{c}^{*+}$ with a mass of $6.34 \mathrm{GeV} / c^{2}$ assumed. 
TABLE V. Branching fractions of semileptonic $P_{1} \rightarrow P_{2}$ transitions calculated in the MD approach and comparison with experimental data. Column $\mathrm{C}$ shows the meson coupled to the weak gauge boson.

\begin{tabular}{|c|c|c|c|c|}
\hline Decay mode & $\mathrm{C}$ & MD result & Data & Notes \\
\hline$\overline{\pi^{+} \rightarrow \pi^{0} e^{+} \nu_{e}}$ & $\overline{\rho^{+}}$ & $(1.0041 \pm 0.0021) \times 10^{-8}$ & $(1.025 \pm 0.034) \times 10^{-8}$ & (a) \\
\hline$K_{L}^{0} \rightarrow K^{ \pm} e^{\mp} \bar{\nu}_{e}\left(\nu_{e}\right)$ & $\rho^{\mp}$ & $(3.4 \pm 0.6) \times 10^{-9}$ & & $(\mathrm{~b}, \mathrm{c}, \mathrm{d})$ \\
\hline$K^{+} \rightarrow \pi^{0} e^{+} \nu_{e}$ & $K^{*+}$ & & $(4.82 \pm 0.06) \%$ & (e) \\
\hline$K^{+} \rightarrow \pi^{0} \mu^{+} \nu_{\mu}$ & $K^{*+}$ & $(3.10 \pm 0.04) \%$ & $(3.18 \pm 0.08) \%$ & \\
\hline$K_{L}^{0} \rightarrow \pi^{ \pm} e^{\mp} \bar{\nu}_{e}\left(\nu_{e}\right)$ & $K^{* \mp}$ & $(40.7 \pm 0.5) \%$ & $(38.78 \pm 0.27) \%$ & (c) \\
\hline$K_{L}^{0} \rightarrow \pi^{ \pm} \mu^{\mp} \bar{\nu}_{\mu}\left(\nu_{\mu}\right)$ & $K^{* \mp}$ & $(26.18 \pm 0.33) \%$ & $(27.17 \pm 0.25) \%$ & (c) \\
\hline$K_{S}^{0} \rightarrow \pi^{ \pm} e^{\mp} \bar{\nu}_{e}\left(\nu_{e}\right)$ & $K^{* \mp}$ & $(7.03 \pm 0.12) \times 10^{-4}$ & $(6.70 \pm 0.07) \times 10^{-4}$ & $(\mathrm{c}, \mathrm{f})$ \\
\hline$K_{S}^{0} \rightarrow \pi^{ \pm} \mu^{\mp} \bar{\nu}_{\mu}\left(\nu_{\mu}\right)$ & $K^{* \mp}$ & $(4.52 \pm 0.08) \times 10^{-4}$ & $(4.69 \pm 0.06) \times 10^{-4}$ & $(\mathrm{c}, \mathrm{f})$ \\
\hline$D^{+} \rightarrow \pi^{0} \ell^{+} \nu_{\ell}$ & $D^{*+}$ & & $(5.7 \pm 2.2) \times 10^{-3}$ & $(\mathrm{e}, \mathrm{g})$ \\
\hline$D^{0} \rightarrow \pi^{-} \ell^{+} \nu_{\ell}$ & $D^{*+}$ & $(4.4 \pm 1.7) \times 10^{-3}$ & $\left(3.8_{-1.0}^{+1.2}\right) \times 10^{-3}$ & $(g)$ \\
\hline$D^{0} \rightarrow K^{-} \mu^{+} \nu_{\mu}$ & $D_{s}^{*+}$ & & $(3.23 \pm 0.19) \%$ & (e) \\
\hline$D^{0} \rightarrow K^{-} e^{+} \nu_{e}$ & $D_{s}^{*+}$ & $(3.33 \pm 0.20) \%$ & $(3.64 \pm 0.20) \%$ & \\
\hline$D^{+} \rightarrow \bar{K}^{0} \mu^{+} \nu_{\mu}$ & $D_{s}^{*+}$ & $(8.3 \pm 0.5) \%$ & $\left(7.0_{-2.0}^{+3.0}\right) \%$ & \\
\hline$D^{+} \rightarrow \bar{K}^{0} e^{+} \nu_{e}$ & $D_{s}^{*+}$ & $(8.6 \pm 0.5) \%$ & $(6.6 \pm 0.9) \%$ & \\
\hline$D_{s}^{+} \rightarrow \eta \ell^{+} \nu_{\ell}$ & $D_{s}^{*+}$ & & $(2.5 \pm 0.7) \%$ & $(e, g)$ \\
\hline$D_{s}^{+} \rightarrow \eta^{\prime} \ell^{+} \nu_{\ell}$ & $D_{s}^{*+}$ & & $(8.7 \pm 3.4) \times 10^{-3}$ & $(\mathrm{e}, \mathrm{g})$ \\
\hline$B^{+} \rightarrow \pi^{0} e^{+} \nu_{e}$ & $B^{*+}$ & $(9.4 \pm 3.1) \times 10^{-5}$ & $<2.2 \times 10^{-3}$ & \\
\hline$B^{+} \rightarrow \pi^{0} \tau^{+} \nu_{\tau}$ & $B^{*+}$ & $(4.9 \pm 1.6) \times 10^{-5}$ & & (b) \\
\hline$B^{0} \rightarrow \pi^{-} \ell^{+} \nu_{\ell}$ & $B^{*+}$ & & $(1.8 \pm 0.6) \times 10^{-4}$ & $(\mathrm{~g}, \mathrm{~h})$ \\
\hline$B^{0} \rightarrow \pi^{-} \tau^{+} \nu_{\tau}$ & $B^{*+}$ & $(9.4 \pm 3.1) \times 10^{-5}$ & & (b) \\
\hline$B^{0} \rightarrow D^{-} \ell^{+} \nu_{\ell}$ & $B_{c}^{*+}$ & & $(1.9 \pm 0.5) \%$ & $(\mathrm{e}, \mathrm{g}, \mathrm{i})$ \\
\hline$B^{0} \rightarrow D^{-} \tau^{+} \nu_{\tau}$ & $B_{c}^{*+}$ & $(4.7 \pm 1.2) \times 10^{-3}$ & & $(b, i)$ \\
\hline$B^{+} \rightarrow \bar{D}^{0} \ell^{+} \nu_{\ell}$ & $B_{c}^{*+}$ & $(2.0 \pm 0.5) \%$ & $(1.6 \pm 0.7) \%$ & $(\mathrm{~g}, \mathrm{i})$ \\
\hline$B^{+} \rightarrow \bar{D}^{0} \tau^{+} \nu_{\tau}$ & $B_{c}^{*+}$ & $(4.9 \pm 1.3) \times 10^{-3}$ & & $(b, \mathrm{i})$ \\
\hline$B_{s}^{0} \rightarrow D_{s}^{-} \ell^{+} \nu_{\ell}$ & $B_{c}^{*+}$ & & $(7.6 \pm 2.4) \%$ & $(e, g, i)$ \\
\hline$B_{s}^{0} \rightarrow D_{s}^{-} \tau^{+} \nu_{\tau}$ & $B_{c}^{*+}$ & $(1.9 \pm 0.6) \%$ & & $(b, \mathrm{i})$ \\
\hline
\end{tabular}

(a) Using $\left|V_{u d}\right|=0.9736 \pm 0.0010$. (b) Not measured yet. (c) The sum of the charge states indicated. (d) Hadronic coupling constant fixed by $\tau^{-} \rightarrow K^{-} K^{0} \nu_{\tau}$. (e) Used to fix normalization. (f) Experimental value was calculated from $K_{L}^{0}$ semileptonic rate and the $K_{S}^{0}$ lifetime assuming $\Delta S=\Delta Q$ [7]. (g) Average of the $e^{+}$and $\mu^{+}$branching fractions. (h) Used to determine $X_{B^{0} \pi^{-} B^{*+}}$. The experimental value is $(1.8 \pm 0.4 \pm 0.3 \pm 0.2) \times 10^{-4}$ [57], where errors are statistical, systematic, end estimated model dependence. We took the liberty of summing the errors quadratically. (i) The existence of $B_{c}^{*+}$ assumed with a mass of $6.34 \mathrm{GeV} / c^{2}$. 
TABLE VI. Branching fractions of the $P_{1} \rightarrow P_{2}+P_{3}$ decay modes in the tree level of MD and comparison with experimental data. Column $\mathrm{C}$ shows the meson coupled to the $W^{+}$boson. Only results that do not contradict existing data are listed.

\begin{tabular}{|c|c|c|c|c|}
\hline Decay mode & $\mathrm{C}$ & Meson dominance result & Data & Notes \\
\hline$\overline{D^{+} \rightarrow \pi^{0} \pi^{+}}$ & $D^{*+}$ & $(4.0 \pm 1.6) \times 10^{-3}$ & $(2.5 \pm 0.7) \times 10^{-3}$ & \\
\hline$D^{0} \rightarrow \pi^{-} K^{+}$ & $D^{*+}$ & $(2.2 \pm 0.9) \times 10^{-4}$ & $(2.9 \pm 1.4) \times 10^{-4}$ & (a) \\
\hline$D^{+} \rightarrow \bar{K}^{0} K^{+}$ & $D_{s}^{*+}$ & $(6.8 \pm 0.4) \times 10^{-3}$ & $(7.2 \pm 1.2) \times 10^{-3}$ & \\
\hline$D^{0} \rightarrow K^{-} \pi^{+}$ & $D_{s}^{*+}$ & $(3.8 \pm 0.2) \%$ & $(3.83 \pm 0.12) \%$ & \\
\hline$D_{s}^{+} \rightarrow \eta \pi^{+}$ & $D_{s}^{*+}$ & $(2.6 \pm 0.7) \%$ & $(2.0 \pm 0.6) \%$ & \\
\hline$B^{+} \rightarrow \pi^{0} \pi^{+}$ & $B^{*+}$ & $(6.8 \pm 2.3) \times 10^{-6}$ & $<1.7 \times 10^{-5}$ & \\
\hline$B^{+} \rightarrow \pi^{0} K^{+}$ & $B^{*+}$ & $(5.1 \pm 1.7) \times 10^{-7}$ & $<1.4 \times 10^{-5}$ & (a) \\
\hline$B^{+} \rightarrow \pi^{0} D_{s}^{+}$ & $B^{*+}$ & $(3.8 \pm 2.1) \times 10^{-5}$ & $<2.0 \times 10^{-4}$ & \\
\hline$B^{0} \rightarrow \pi^{-} \pi^{+}$ & $B^{*+}$ & $(1.3 \pm 0.4) \times 10^{-5}$ & $<2.0 \times 10^{-5}$ & \\
\hline$B^{0} \rightarrow \pi^{-} K^{+}$ & $B^{*+}$ & $(9.9 \pm 3.3) \times 10^{-7}$ & $<1.7 \times 10^{-5}$ & (a) \\
\hline$B^{0} \rightarrow \pi^{-} D_{s}^{+}$ & $B^{*+}$ & $(7.4 \pm 4.1) \times 10^{-5}$ & $<2.8 \times 10^{-4}$ & \\
\hline$B^{+} \rightarrow \bar{D}^{0} D_{s}^{+}$ & $B_{c}^{*+}$ & $(1.9 \pm 1.0) \%$ & $(1.7 \pm 0.6) \%$ & (b) \\
\hline$B^{0} \rightarrow D^{-} \pi^{+}$ & $B_{c}^{*+}$ & $(3.6 \pm 0.9) \times 10^{-3}$ & $(3.0 \pm 0.4) \times 10^{-3}$ & (b) \\
\hline$B^{0} \rightarrow D^{-} D_{s}^{+}$ & $B_{c}^{*+}$ & $(18 \pm 10) \times 10^{-3}$ & $(7 \pm 4) \times 10^{-3}$ & (b) \\
\hline$B_{s}^{0} \rightarrow D_{s}^{-} \pi^{+}$ & $B_{c}^{*+}$ & $(1.4 \pm 0.5) \%$ & $<12 \%$ & (b) \\
\hline
\end{tabular}

(a) Doubly Cabibbo suppressed mode. (b) Existence of $B_{c}^{*+}$ with a mass of $6.34 \mathrm{GeV} / c^{2}$ assumed.

TABLE VII. Branching fractions of the $P_{1} \rightarrow P_{2}+P_{3}$ decay modes in the tree level of MD and comparison with experimental data. Column $\mathrm{C}$ shows the meson coupled to the $W^{+}$boson. Only results that contradict data are listed.

\begin{tabular}{llccc}
\hline \hline Decay mode & $\mathrm{C}$ & Meson dominance result & Data & Notes \\
\hline$K^{+} \rightarrow \pi^{0} \pi^{+}$ & $K^{*+}$ & $(86.3 \pm 1.1) \%$ & $(21.16 \pm 0.14) \%$ & $(68.61 \pm 0.28) \%$ \\
$K_{S}^{0} \rightarrow \pi^{-} \pi^{+}$ & $K^{*+}$ & $(2.52 \pm 0.03) \%$ & $(31.39 \pm 0.28) \%$ \\
$K_{S}^{0} \rightarrow \pi^{0} \pi^{0}$ & none & 0 & $(1.52 \pm 0.11) \times 10^{-3}$ & $(8.4 \pm 2.2) \times 10^{-4}$ \\
$D^{0} \rightarrow \pi^{-} \pi^{+}$ & $D^{*+}$ & $(3.1 \pm 1.2) \times 10^{-3}$ & $(2.74 \pm 0.29) \%$ \\
$D^{0} \rightarrow \pi^{0} \pi^{0}$ & none & 0 & $(2.11 \pm 0.21) \%$ \\
$D^{+} \rightarrow \bar{K}^{0} \pi^{+}$ & $D_{s}^{*+}$ & $(9.8 \pm 0.6) \%$ & $(4.33 \pm 0.27) \times 10^{-3}$ & $(1.3 \pm 0.4) \times 10^{-3}$ \\
$D^{0} \rightarrow \bar{K}^{0} \pi^{0}$ & none & 0 & $(4.9 \pm 1.8) \%$ & \\
$D^{0} \rightarrow K^{-} K^{+}$ & $D_{s}^{*+}$ & $(2.66 \pm 0.16) \times 10^{-3}$ & $(5.3 \pm 0.5) \times 10^{-3}$ & $(\mathrm{a})$ \\
$D^{0} \rightarrow \bar{K}^{0} K^{0}$ & none & 0 & \\
$D_{s}^{+} \rightarrow \eta^{\prime} \pi^{+}$ & $D_{s}^{*+}$ & $(1.9 \pm 0.8) \%$ & $(3.7 \pm 1.0) \times 10^{-3}$ & \\
$B^{+} \rightarrow \bar{D}^{0} \pi^{+}$ & $B_{c}^{*+}$ &
\end{tabular}

(a) Existence of $B_{c}^{*+}$ with a mass of $6.34 \mathrm{GeV} / c^{2}$ assumed. 
TABLE VIII. Branching fractions in the tree level of MD of the $P_{1} \rightarrow P_{2}+P_{3}$ decay modes that have not yet been observed. Only the modes with branching fractions greater than $\times 10^{-4}$ listed. Column $\mathrm{C}$ shows the meson coupled to the $W^{+}$boson.

\begin{tabular}{llcl}
\hline \hline Decay mode & $\mathrm{C}$ & Tree diagram of MD & Notes \\
\hline$D^{+} \rightarrow \pi^{0} K^{+}$ & $D^{*+}$ & $(2.9 \pm 1.1) \times 10^{-4}$ & $(\mathrm{a})$ \\
$D^{+} \rightarrow \eta K^{+}$ & $D^{*+}$ & $(5.2 \pm 1.7) \times 10^{-4}$ & $(\mathrm{a})$ \\
$D^{+} \rightarrow \eta^{\prime} K^{+}$ & $D^{*+}$ & $<5.7 \times 10^{-4}$ & $(\mathrm{a}, \mathrm{b})$ \\
$D_{s}^{+} \rightarrow \eta K^{+}$ & $D_{s}^{*+}$ & $(1.8 \pm 0.5) \times 10^{-3}$ & \\
$D_{s}^{+} \rightarrow \eta^{\prime} K^{+}$ & $D_{s}^{*+}$ & $(1.3 \pm 0.5) \times 10^{-3}$ & $(\mathrm{a}, \mathrm{c}, \mathrm{d})$ \\
$D_{s}^{+} \rightarrow K^{0} K^{+}$ & $D^{*+}$ & $\left(2.8 \pm 0.6 \times 10^{-4}\right.$ & $(\mathrm{a}, \mathrm{e})$ \\
$B^{+} \rightarrow \bar{D}^{0} K^{+}$ & $B_{c}^{*+}$ & $(4.0 \pm 1.4) \times 10^{-4}$ & $(\mathrm{a}, \mathrm{e}, \mathrm{f})$ \\
$B^{+} \rightarrow \bar{D}^{0} D^{+}$ & $B_{c}^{*+}$ & $(2.7 \pm 0.7) \times 10^{-4}$ & $(\mathrm{a}, \mathrm{e})$ \\
$B^{0} \rightarrow D^{-} K^{+}$ & $B_{c}^{*+}$ & $(3.8 \pm 1.3) \times 10^{-4}$ & $(\mathrm{a}, \mathrm{e}, \mathrm{f})$ \\
$B^{0} \rightarrow D^{-} D^{+}$ & $B_{c}^{*+}$ & $(1.1 \pm 0.4) \times 10^{-3}$ & $(\mathrm{a})$ \\
$B_{s}^{0} \rightarrow D_{s}^{-} K^{+}$ & $B_{c}^{*+}$ & $(1.5 \pm 0.6) \times 10^{-3}$ & $(\mathrm{a}, \mathrm{e}, \mathrm{f})$ \\
$B_{s}^{0} \rightarrow D_{s}^{-} D^{+}$ & $B_{c}^{*+}$ & $(7.4 \pm 4.0) \%$ & $(\mathrm{e})$ \\
$B_{s}^{0} \rightarrow D_{s}^{-} D_{s}^{+}$ & $B_{c}^{*+}$ & \\
\hline \hline
\end{tabular}

(a) Doubly Cabibbo suppressed mode. (b) Using $B\left(D^{+} \rightarrow \eta^{\prime} \pi^{+}\right)<9 \times 10^{-3}$. (c) Using $B\left(D_{s}^{+} \rightarrow K^{0} \pi^{+}\right)<8 \times 10^{-3}$. (d) This mode is experimentally indistinguishable from $D_{s}^{+} \rightarrow \bar{K}^{0} K^{+}$and represents a negligible background to it. (e) Existence of $B_{c}^{*+}$ with a mass of $6.34 \mathrm{GeV} / c^{2}$ assumed. (f) $D^{+}$decay constant taken from [15. 
TABLE IX. Branching fractions of the $P_{1} \rightarrow P_{2}+V(A)$ decay modes in the tree level of MD and comparison with experimental data. Column $\mathrm{C}$ shows the meson coupled to the $W^{+}$boson. Only results that do not contradict data are listed.

\begin{tabular}{llccc}
\hline \hline Decay mode & $\mathrm{C}$ & Meson dominance result & Data & Notes \\
\hline$D_{s}^{+} \rightarrow \eta^{\prime} \rho^{+}$ & $D_{s}^{*+}$ & $(11.9 \pm 4.6) \%$ & $(12 \pm 4) \%$ & \\
$B^{+} \rightarrow \pi^{0} \rho^{+}$ & $B^{*+}$ & $(1.2 \pm 0.4) \times 10^{-5}$ & $<7.7 \times 10^{-5}$ & \\
$B^{+} \rightarrow \pi^{0} K^{*+}$ & $B^{*+}$ & $(7.4 \pm 2.5) \times 10^{-7}$ & $<9.9 \times 10^{-5}$ & $(\mathrm{a})$ \\
$B^{+} \rightarrow \pi^{0} D_{s}^{*+}$ & $B^{*+}$ & $(6 \pm 5) \times 10^{-5}$ & $<3.3 \times 10^{-4}$ & $(\mathrm{~b})$ \\
$B^{+} \rightarrow \pi^{0} a_{1}^{+}$ & $B^{*+}$ & $(2.0 \pm 0.7) \times 10^{-5}$ & $<1.7 \times 10^{-3}$ & \\
$B^{0} \rightarrow \pi^{-} \rho^{+}$ & $B^{*+}$ & $(2.4 \pm 0.8) \times 10^{-5}$ & $<8.8 \times 10^{-5}$ & $(\mathrm{c})$ \\
$B^{0} \rightarrow \pi^{-} K^{*+}$ & $B^{*+}$ & $(1.4 \pm 0.5) \times 10^{-6}$ & $<7.2 \times 10^{-5}$ & $(\mathrm{a})$ \\
$B^{0} \rightarrow \pi^{-} D_{s}^{*+}$ & $B^{*+}$ & $(1.1 \pm 1.0) \times 10^{-4}$ & $<5 \times 10^{-4}$ & $(\mathrm{~b})$ \\
$B^{0} \rightarrow \pi^{-} a_{1}^{+}$ & $B^{*+}$ & $(3.8 \pm 1.3) \times 10^{-5}$ & $<4.9 \times 10^{-4}$ & $(\mathrm{~d})$ \\
$B^{+} \rightarrow \eta D_{s}^{*+}$ & $B^{*+}$ & $(7 \pm 6) \times 10^{-4}$ & $<8 \times 10^{-4}$ & $(\mathrm{~b})$ \\
$B^{+} \rightarrow \bar{D}^{0} D_{s}^{*+}$ & $B_{c}^{*+}$ & $(2.0 \pm 1.7) \%$ & $(1.2 \pm 1.0) \%$ & $(\mathrm{~b}, \mathrm{e})$ \\
$B^{+} \rightarrow \bar{D}^{0} a_{1}^{+}$ & $B_{c}^{*+}$ & $(9.8 \pm 2.6) \times 10^{-3}$ & $(5 \pm 4) \times 10^{-3}$ & $(\mathrm{e})$ \\
$B^{0} \rightarrow D^{-} \rho^{+}$ & $B_{c}^{*+}$ & $(6.3 \pm 1.7) \times 10^{-3}$ & $(7.8 \pm 1.4) \times 10^{-3}$ & $(\mathrm{e})$ \\
$B^{0} \rightarrow D^{-} a_{1}^{+}$ & $B_{c}^{*+}$ & $(9.4 \pm 2.5) \times 10^{-3}$ & $(6.0 \pm 3.3) \times 10^{-3}$ & $(\mathrm{e})$ \\
\hline \hline
\end{tabular}

(a) Doubly Cabibbo suppressed mode. (b) Using $\left(w_{D_{s}^{*}}\left|V_{c s}\right|\right)^{2}=39 \pm 31$, as determined from $B\left(B^{0} \rightarrow D^{-} D_{s}^{*+}\right)=(2.0 \pm 1.5) \%$. (c) Experimental value includes also the $\pi^{+} \rho^{-}$mode. (d) Experimental value includes also the $\pi^{+} a_{1}^{-}$mode. (e) Existence of $B_{c}^{*+}$ with a mass of 6.34 $\mathrm{GeV} / c^{2}$ assumed.

TABLE X. Branching fractions of the $P_{1} \rightarrow P_{2}+V(A)$ decay modes in the tree level of MD and comparison with experimental data. Column $\mathrm{C}$ shows the meson coupled to the $W^{+}$boson. Only results that contradict existing data are listed.

\begin{tabular}{llccc}
\hline \hline Decay mode & $\mathrm{C}$ & Meson dominance result & Data & Notes \\
\hline$D^{+} \rightarrow \bar{K}^{0} \rho^{+}$ & $D_{s}^{*+}$ & $(11.7 \pm 0.7) \%$ & $(6.6 \pm 2.5) \%$ & \\
$D^{0} \rightarrow \bar{K}^{0} \rho^{0}$ & none & 0 & $(1.20 \pm 0.17) \%$ & \\
$D^{0} \rightarrow K^{-} \rho^{+}$ & $D_{s}^{*+}$ & $(4.57 \pm 0.27) \%$ & $(3.0 \pm 1.4) \%$ & \\
$D^{+} \rightarrow \bar{K}^{0} K^{*+}$ & $D_{s}^{*+}$ & $(0.6 \pm 0.4) \%$ & $(3.5 \pm 0.8) \times 10^{-3}$ & \\
$D^{0} \rightarrow K^{-} K^{*+}$ & $D_{s}^{*+}$ & $(2.20 \pm 0.14) \times 10^{-3}$ & $(7.1 \pm 1.7) \%$ & \\
$D^{+} \rightarrow \bar{K}^{0} a_{1}^{+}$ & $D_{s}^{*+}$ & $(3.77 \pm 0.22) \%$ & $(10.3 \pm 3.2) \%$ & \\
$D^{0} \rightarrow K^{-} a_{1}^{+}$ & $D_{s}^{*+}$ & $(1.5 \pm 0.1) \%$ & $(1.34 \pm 0.18) \%$ & $(\mathrm{a})$ \\
$D_{s}^{+} \rightarrow \eta \rho^{+}$ & $D_{s}^{*+}$ & $(3.3 \pm 0.9) \%$ & & \\
$B^{+} \rightarrow \bar{D}^{0} \rho^{+}$ & $B_{c}^{*+}$ & $(0.7 \pm 0.2) \%$ & & \\
\hline \hline
\end{tabular}

(a) Existence of $B_{c}^{*+}$ with a mass of $6.34 \mathrm{GeV} / c^{2}$ assumed. 
TABLE XI. Selected $B_{c}^{+}$decays: MD predictions for branching ratios and their comparison with those of various models.

\begin{tabular}{ccccccc}
\hline \hline Branching ratio & MD & PQCD [9] & BS [58] & ISGW [59] & BSW [59] & BSW [60] \\
\hline$B_{c}^{+} \rightarrow \eta_{c}+\ell^{+} \nu_{\ell} / \pi^{+}$ & 5.0 & & 4.3 & 4.0 & & 4.1 \\
$\tau^{+} \nu_{\tau} / \pi^{+}$ & 1.1 & & & & & \\
$K^{+} / \pi^{+}$ & 0.075 & 0.068 & 0.078 & 0.074 & & 0.078 \\
$\rho^{+} / \pi^{+}$ & 2.3 & 3.0 & 2.6 & 2.4 & & 2.6 \\
$K^{*+} / \pi^{+}$ & 0.10 & 0.09 & 0.14 & 0.12 & & 0.14 \\
$a_{1}^{+} / \pi^{+}$ & 2.6 & & & & & \\
$K_{1}^{+} / \pi^{+}$ & 0.18 & & & & & \\
$B_{c}^{+} \rightarrow B_{s}+\ell^{+} \nu_{\ell} / \pi^{+}$ & 0.38 & & 0.36 & 0.26 & 0.25 & 0.27 \\
$K^{+} / \pi^{+}$ & 0.064 & & 0.072 & 0.075 & 0.070 & 0.073 \\
$\rho^{+} / \pi^{+}$ & 0.45 & & 0.77 & 0.46 & 0.40 & 0.60 \\
\hline \hline
\end{tabular}

PQCD: using perturbative QCD framework proposed in [61]; BS: Bethe-Salpeter description of the meson wave functions and the hadronic matrix elements; ISGW: model of Isgur, Scora, Grinstein, and Wise [62]; BSW: model of Wirbel, Stech, and Bauer [63].

TABLE XII. Branching fractions of the flavor changing "weak neutral current" modes $P_{1} \rightarrow P_{2}+\ell^{+} \ell^{-}$calculated in the MD approach assuming the dominant role of the $a_{1}$ resonance. Column $\mathrm{C}$ shows the mesons coupled to $W^{+}$.

\begin{tabular}{|c|c|c|c|c|}
\hline Decay mode & $\mathrm{C}$ & MD result & Other predictions & Data \\
\hline$\overline{K^{+} \rightarrow \pi^{+} e^{+} e^{-}}$ & $K^{+}, a_{1}^{+}$ & $\approx 3.1 \times 10^{-7}(\mathrm{a})$ & & $(2.74 \pm 0.23) \times 10^{-7}$ \\
\hline$K^{+} \rightarrow \pi^{+} \mu^{+} \mu^{-}$ & $K^{+}, a_{1}^{+}$ & $(6.2 \pm 0.5) \times 10^{-8}(\mathrm{~b})$ & $\left(6.2_{-0.6}^{+0.8}\right) \times 10^{-8}$ & $<2.3 \times 10^{-7}$ \\
\hline$D^{+} \rightarrow \pi^{+} e^{+} e^{-}$ & $D^{+}, a_{1}^{+}$ & $(3.9 \pm 0.9) \times 10^{-7}(\mathrm{c})$ & $<10^{-8}$ & $<6.6 \times 10^{-5}$ \\
\hline$D^{+} \rightarrow \pi^{+} \mu^{+} \mu^{-}$ & $D^{+}, a_{1}^{+}$ & $(3.9 \pm 0.9) \times 10^{-7}(\mathrm{c})$ & $<10^{-8}$ & $<1.8 \times 10^{-5}$ \\
\hline$D_{s}^{+} \rightarrow \pi^{+} \mu^{+} \mu^{-}$ & $D_{s}^{+}, a_{1}^{+}$ & $(1.0 \pm 0.5) \times 10^{-5}(\mathrm{~d})$ & & $<4.3 \times 10^{-4}$ \\
\hline
\end{tabular}

(a) Using the $a_{1} \rho \pi$ coupling constant determined from $\Gamma_{a_{1}} \approx 400 \mathrm{MeV}$. (b) Normalized by the $K^{+} \rightarrow \pi^{+} e^{+} e^{-}$experimental branching fraction. (c) Using the $a_{1} \rho \pi$ coupling constant determined from $K^{+} \rightarrow \pi^{+} e^{+} e^{-}$and the lattice calculation [15] result for the $D^{+}$decay constant. (d) Using the $a_{1} \rho \pi$ coupling constant determined from $K^{+} \rightarrow \pi^{+} e^{+} e^{-}$and the $D_{s}^{+}$decay constant from the experimental branching fraction of $D_{s}^{+} \rightarrow \mu^{+} \nu_{\mu}$. 


\section{FIGURES}

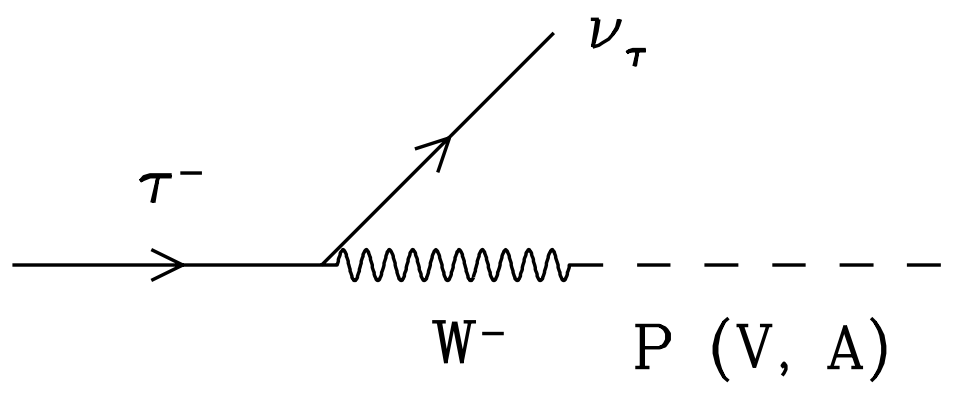

FIG. 1. Matrix element of the $\tau^{-}$decay to neutrino and a pseudoscalar, vector, or axial-vector meson. 


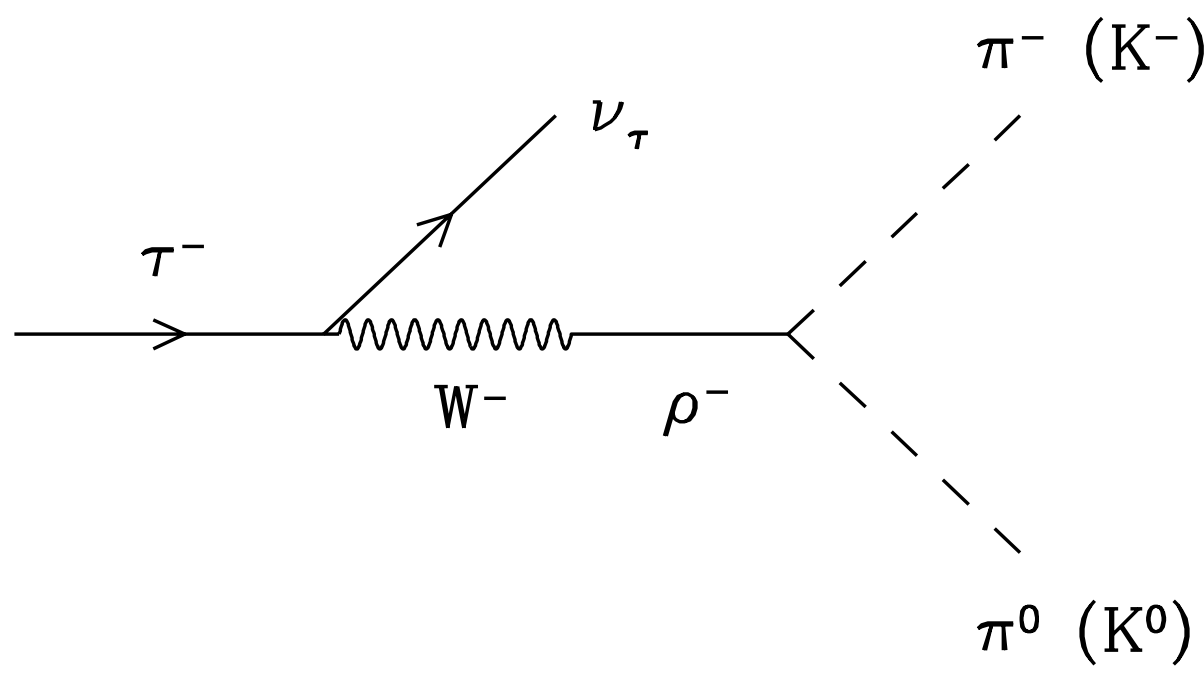

FIG. 2. Matrix element of the decays $\tau^{-} \rightarrow \pi^{-} \pi^{0} \nu_{\tau}$ and $\tau^{-} \rightarrow K^{-} K^{0} \nu_{\tau}$. 


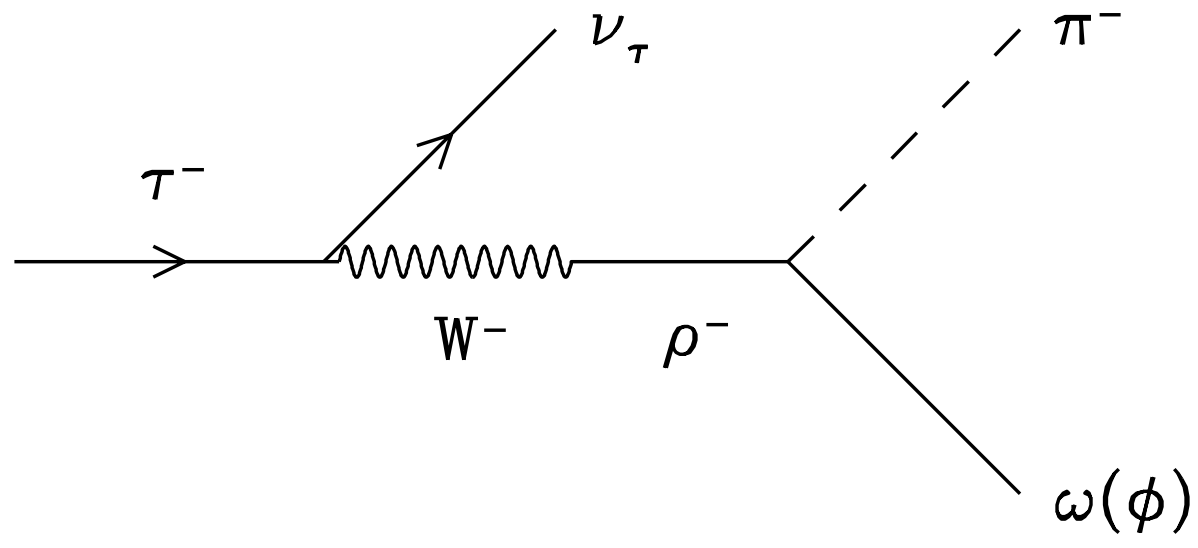

FIG. 3. Matrix element of the decay $\tau^{-} \rightarrow \omega(\phi) \pi^{-} \nu_{\tau}$. 


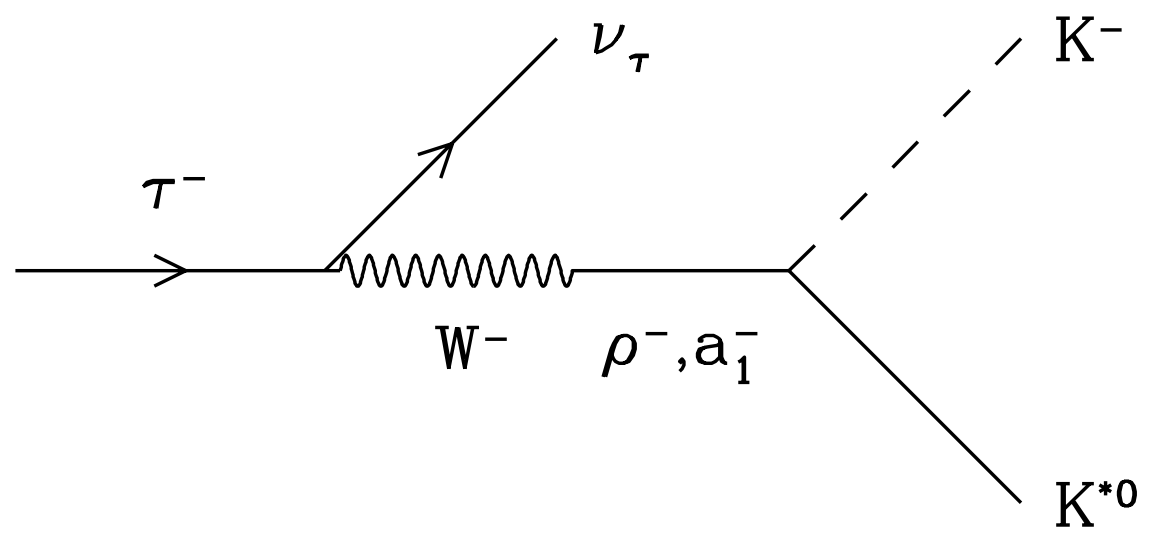

FIG. 4. Two diagrams that contribute to decay $\tau^{-} \rightarrow K^{* 0} K^{-} \nu_{\tau}$, one with $\rho^{-}$, the other with $a_{1}^{-}$in the intermediate state. 


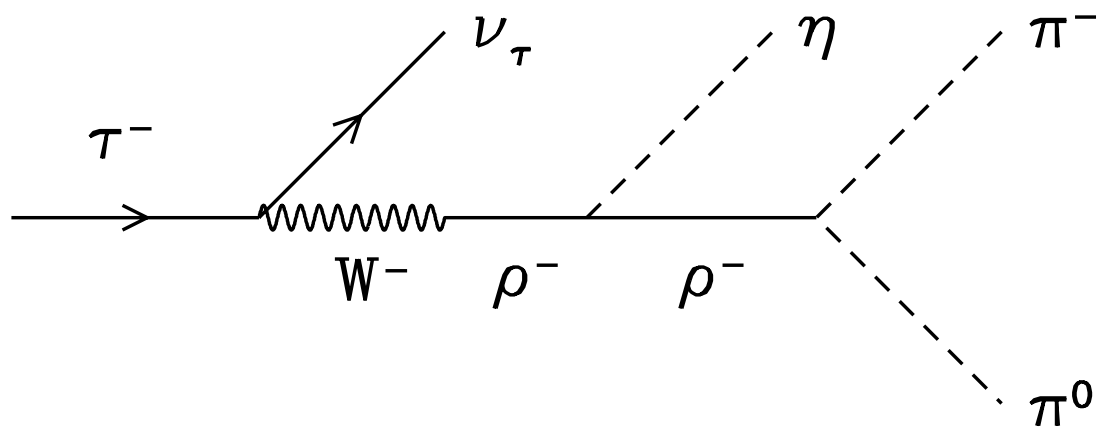

FIG. 5. Matrix element of the decay $\tau^{-} \rightarrow \eta \pi^{-} \pi^{0} \nu_{\tau}$. 


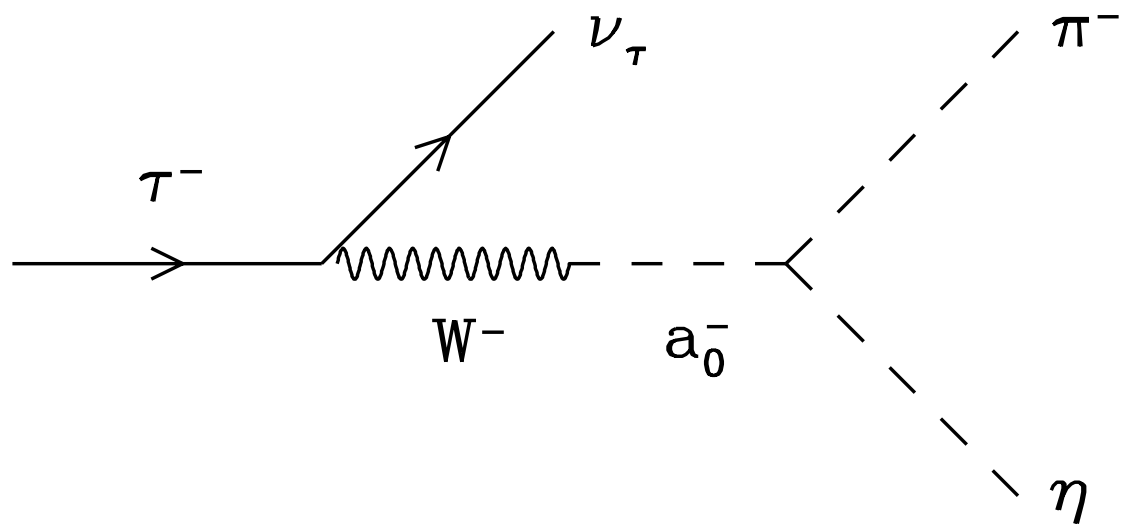

FIG. 6. Matrix element of the decay $\tau^{-} \rightarrow \eta \pi^{-} \nu_{\tau}$. 


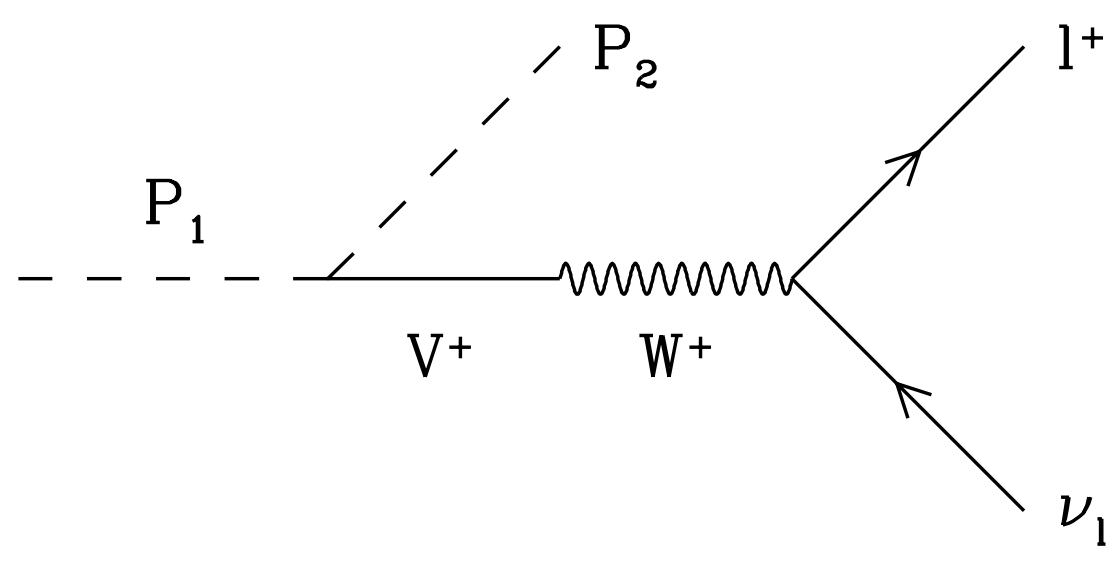

FIG. 7. Generic Feynman diagram of $P_{1} \rightarrow P_{2} \ell^{+} \nu_{\ell}$ decays. 


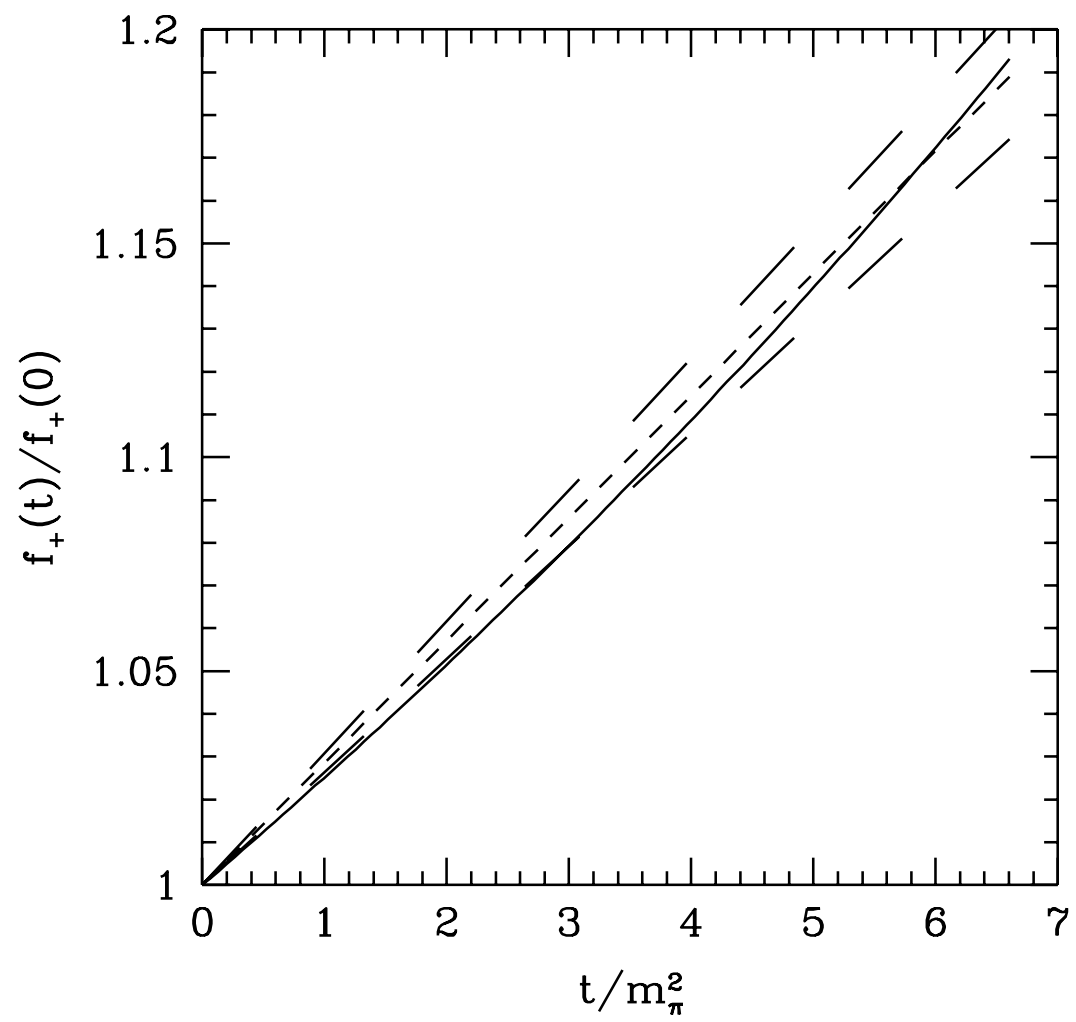

FIG. 8. $K_{e 3}^{+}$form factor $f_{+}(t)$ : Meson dominance (solid), linear parametrization used by experimentalists to fit data with limits coming from the experimental error of the slope parameter (dashed). 


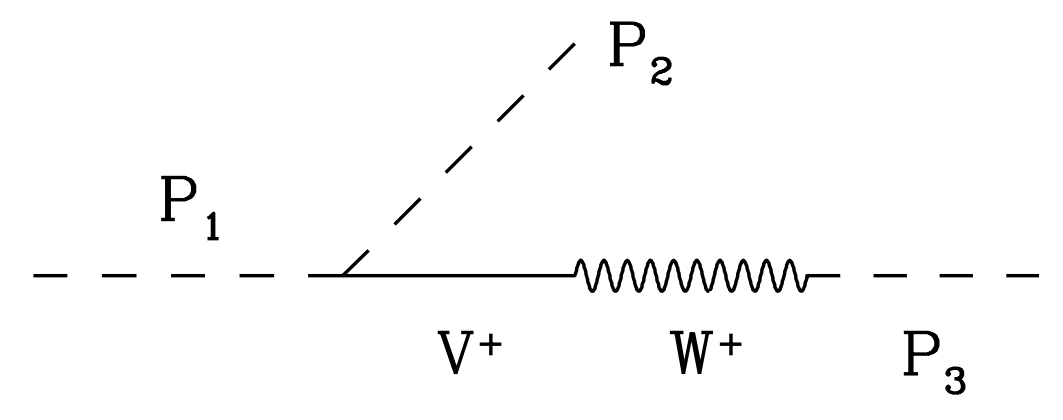

FIG. 9. Generic Feynman diagram of $P_{1} \rightarrow P_{2}+P_{3}$ decays. 


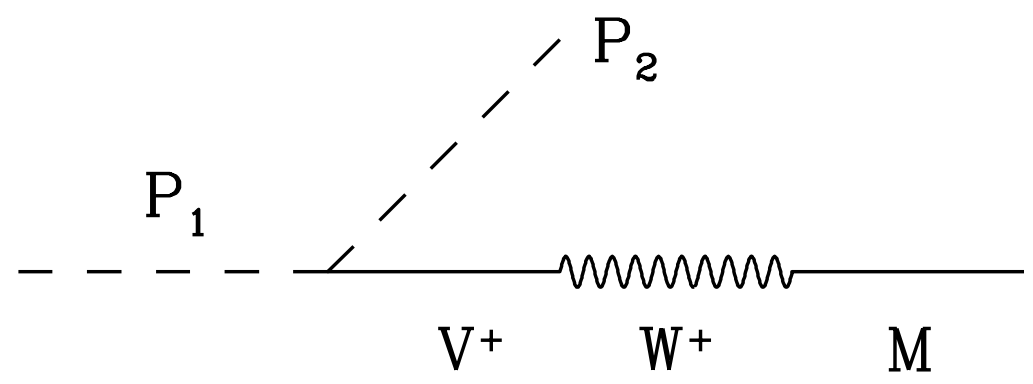

FIG. 10. Generic Feynman diagram of $P_{1} \rightarrow P_{2}+M$ decays. $M$ stands for the outgoing vector or axial-vector meson. 


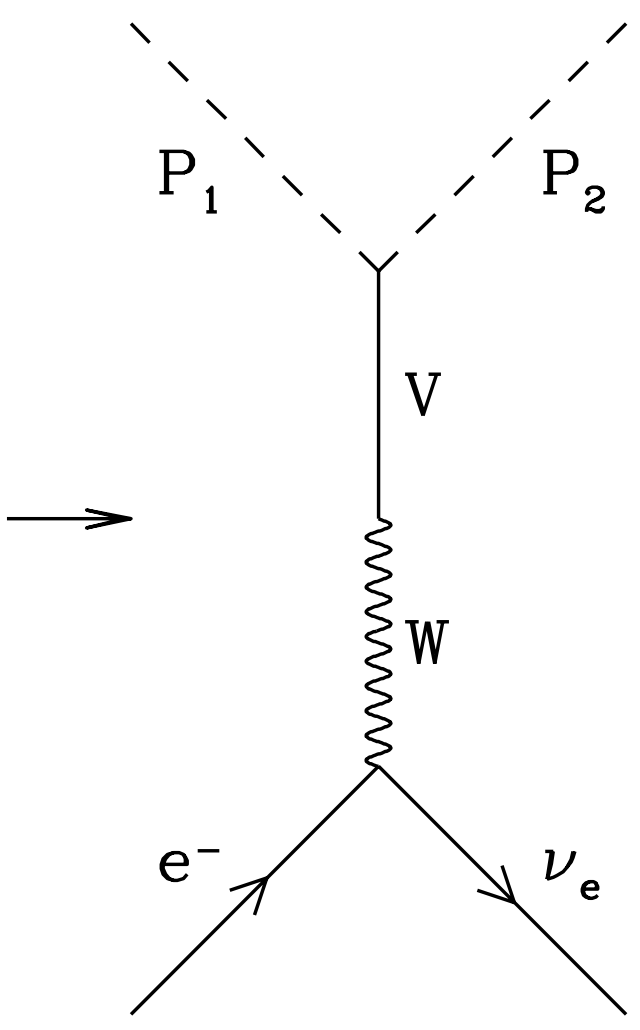

FIG. 11. Matrix element of the reaction $P_{1}^{+}+e^{-} \rightarrow P_{2}^{-}+\nu_{e}$. 


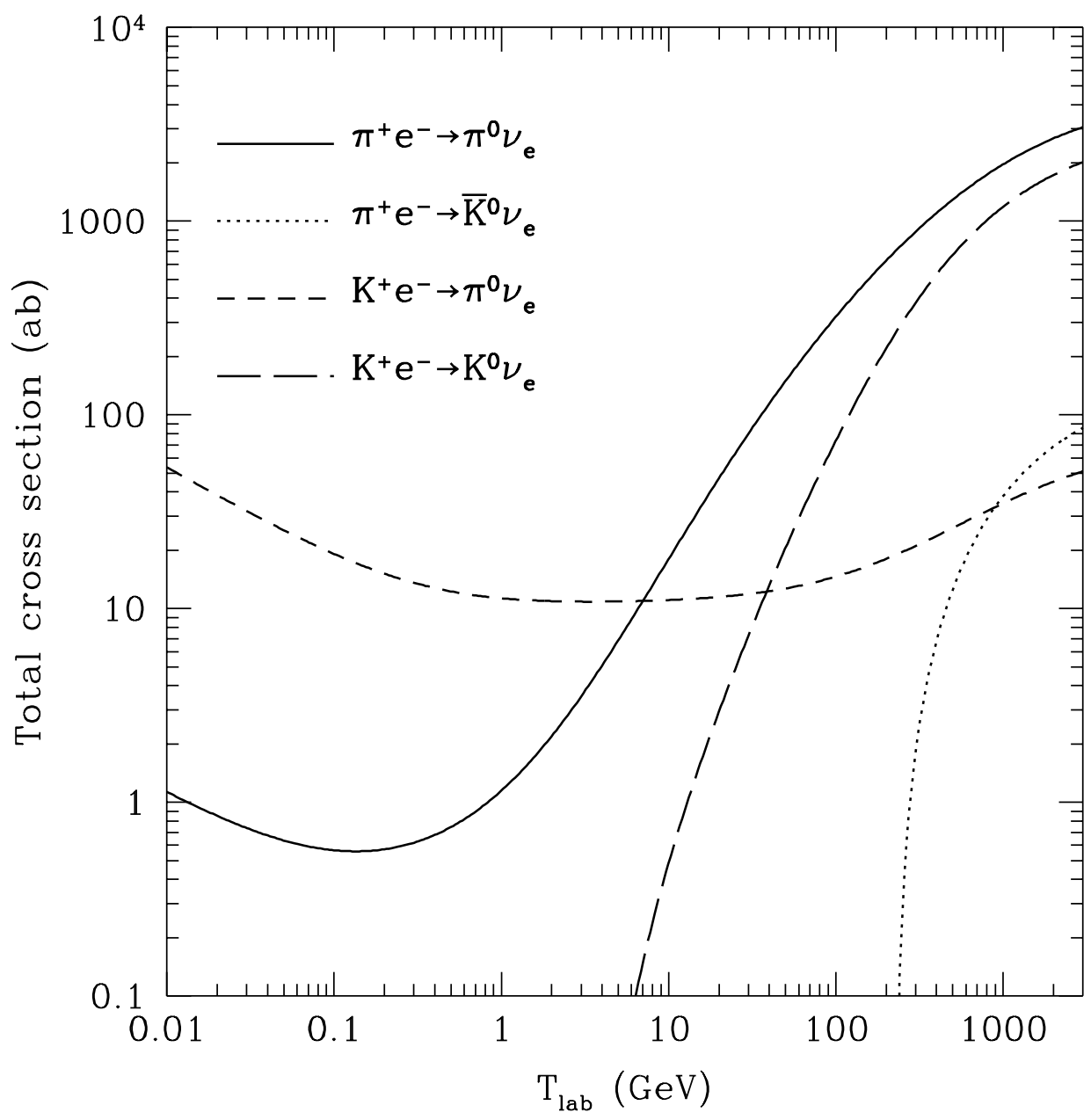

FIG. 12. Total cross section in attobarns $\left(1 \mathrm{ab}=10^{-42} \mathrm{~cm}^{2}\right)$ of the reactions of positive pions and kaons with target electrons as a function of the laboratory kinetic energy. 


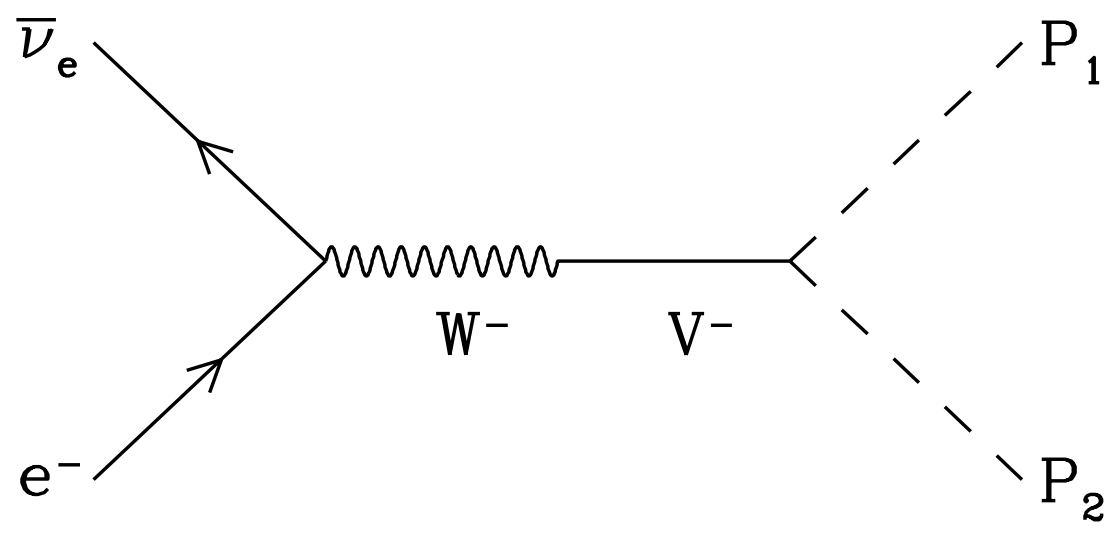

FIG. 13. Matrix element of the reaction $\bar{\nu}_{e}+e^{-} \rightarrow P_{1}+P_{2}$. 


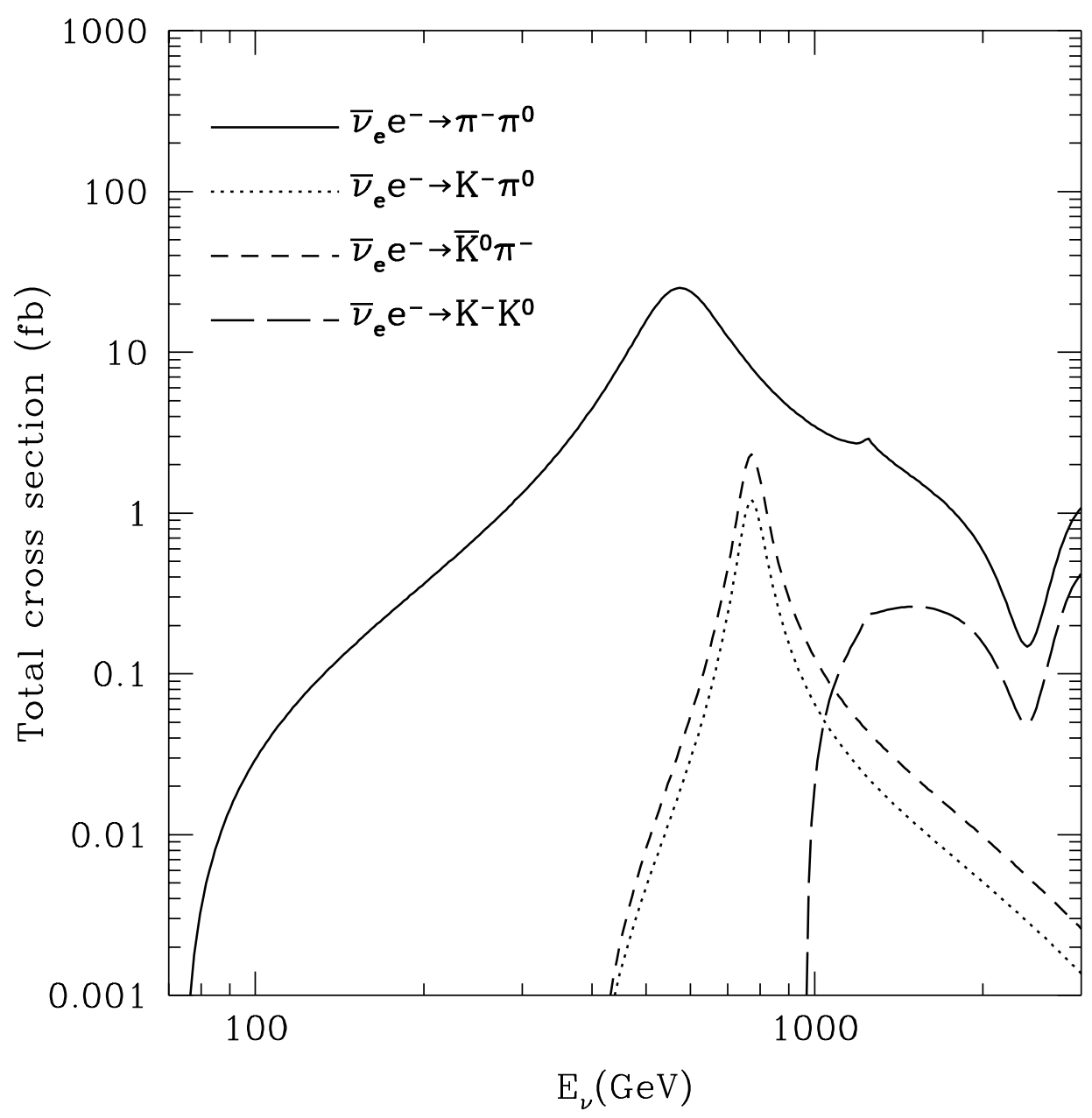

FIG. 14. Total cross section in femtobarns $\left(1 \mathrm{fb}=10^{-39} \mathrm{~cm}^{2}\right)$ of two-meson production in reactions of the electron antineutrino with target electrons as a function of antineutrino energy. 


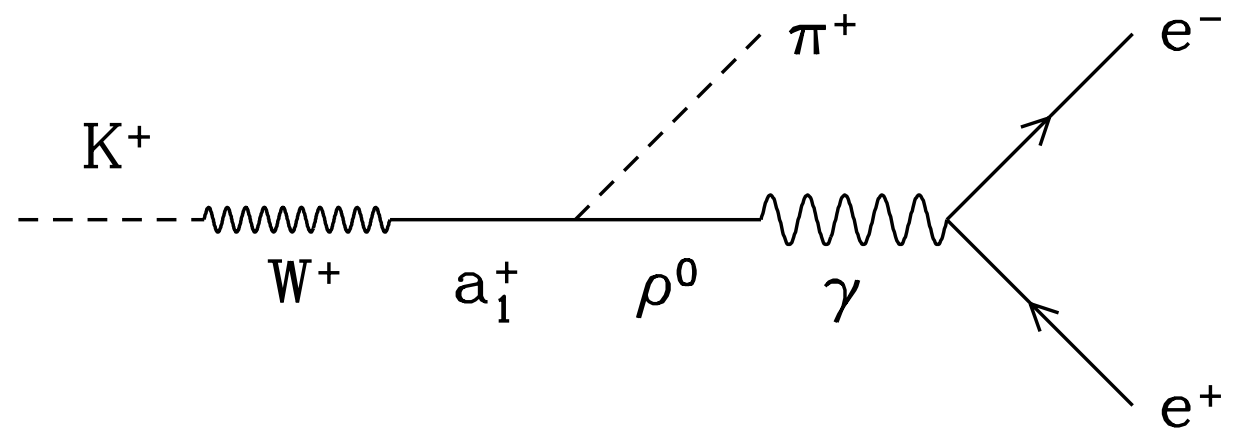

FIG. 15. Matrix element of the decay $K^{+} \rightarrow \pi^{+} e^{+} e^{-}$in the meson dominance approach. 


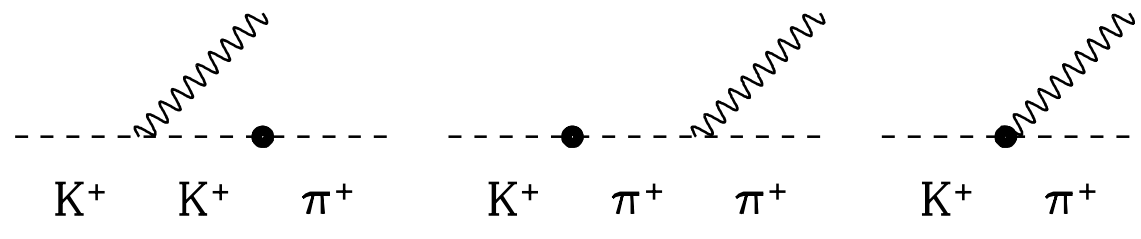

FIG. 16. Three contributions to the matrix element of the decay $K^{+} \rightarrow \pi^{+} \gamma\left(\gamma^{*}\right)$ related to Lagrangians (5.5) and (5.6). 


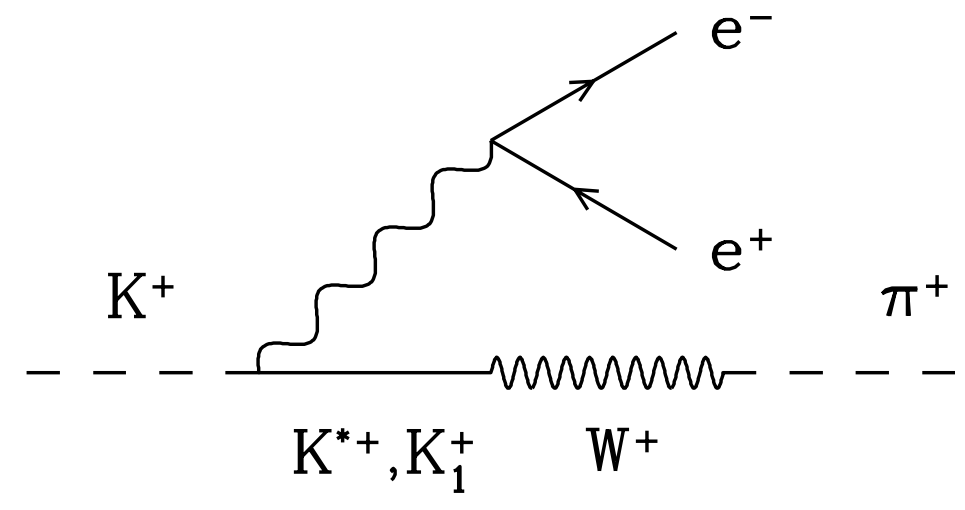

FIG. 17. Other two possible MD Feynman diagrams for $K^{+} \rightarrow \pi^{+} e^{+} e^{-}$. 


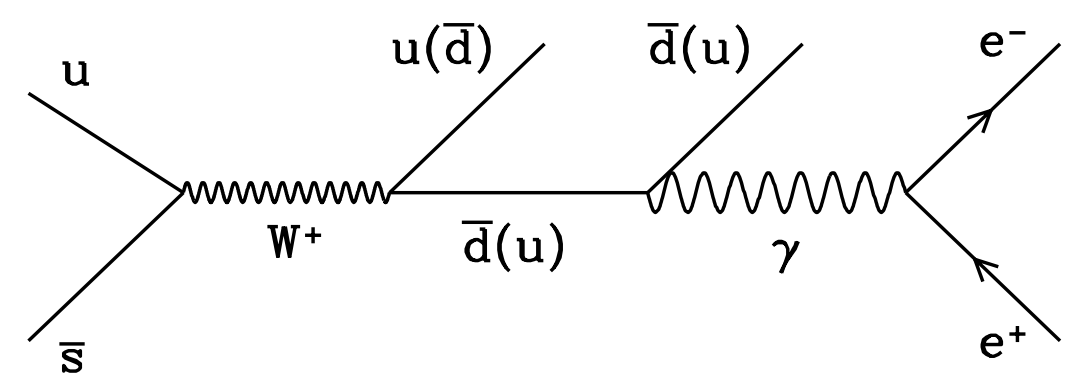

(a)

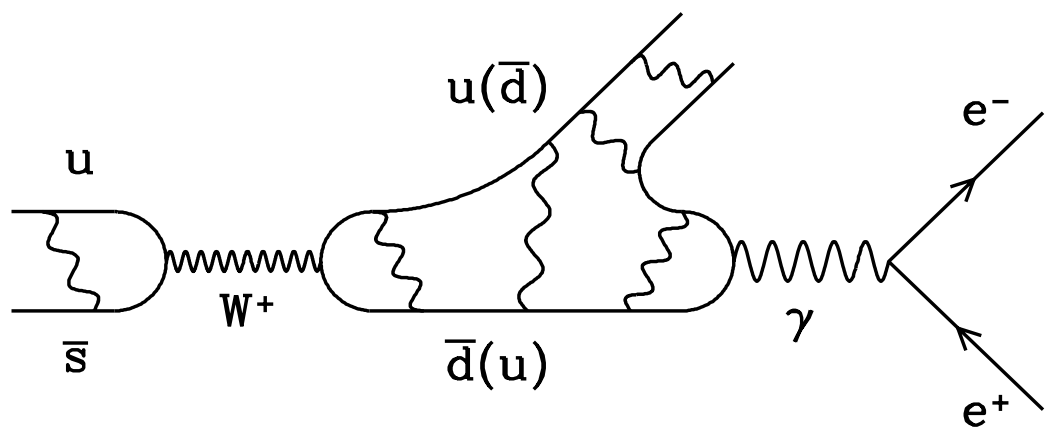

(b)

FIG. 18. Selected quark diagrams of the $K^{+} \rightarrow \pi^{+} e^{+} e^{-}$decay mode. (a) Two (differing by $u \leftrightarrow \bar{d}$ ) of possible electroweak diagrams; (b) After the strong interactions are switched on, the previous diagrams develop into those that provide the most important contributions, like the one shown here. Unlabeled wavy curves represent gluons. 


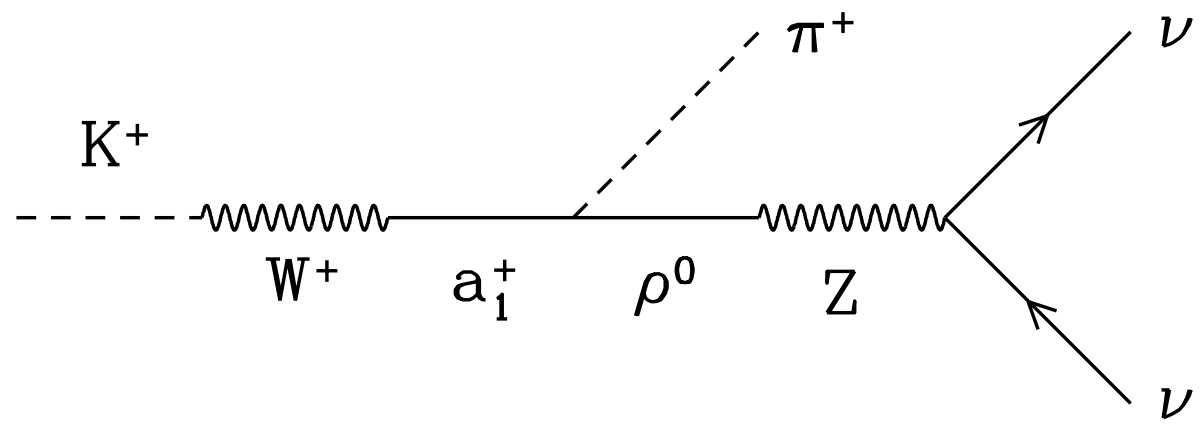

FIG. 19. Long-distance part of the matrix element of the CP conserving decay $K^{+} \rightarrow \pi^{+} \nu \bar{\nu}$. 